\title{
Pd(II)-catalyzed diastereoselective and enantioselective domino cyclization/cycloaddition reactions of alkenyl oximes for polycyclic heterocycles with four chiral stereogenic centers
}

\author{
Mohamed A. Abozeid, Shinobu Takizawa* and Hiroaki Sasai \\ The Institute of Scientific and Industrial Research (ISIR), Osaka University, Mihogaoka, Ibaraki-shi, Osaka 567-0047, Japan.
}

\section{ARTICLE INFO}

\section{ABSTRACT}

\section{Article history:}

Received

Received in revised form

Accepted

Available online

Keywords:

Stereoselective reaction

Domino reaction

Cyclization

Cycloaddition

Isoxazolidine
Diastereoselective and enantioselective domino cyclization/cycloaddition reactions of alkenyl oximes were established using a Pd(II)-( $R$ )-Tol-SDP complex and triflic acid. The present process gave polycyclic heterocycles with four chiral stereogenic centers in almost quantitative yields and high stereoselectivities (up to $70 \%$ ee, exo/endo $=97 / 3$ ).

2009 Elsevier Ltd. All rights reserved.
The development of a facile construction of polycyclic heterocycles is a subject of intensive research because of their potential use in medicinal chemistry. ${ }^{1,2}$ Among them, domino cyclization has become a powerful strategy for the formation of two or more rings in a single operation. In the domino cyclization, multiple chiral centers can often be formed with high stereoselectivities. ${ }^{2}$ Nitrones derived from the reaction of oximes and alkenes are widely used 1,3-dipoles that endure cycloadditions with alkenes affording versatile isoxazolidine derivatives. ${ }^{3}$ In 1994, Grigg et al. developed Pd(II)-catalyzed cyclization/cycloaddition cascade reaction of alkenyl oximes, effectively furnishing isoxazolidines with multiple stereocenters. ${ }^{4}$ Despite the potential of this transformation, no enantioselective domino cyclization process has been reported. ${ }^{5}$ Herein, we present the first enantioselective protocol of a cyclization/cycloaddition sequence of alkenyl oxime $(E)-\mathbf{1}$ with enedione 2 catalyzed by a Pd(II)-(R)-Tol-SDP complex and triflic acid (TfOH) (Scheme 1).

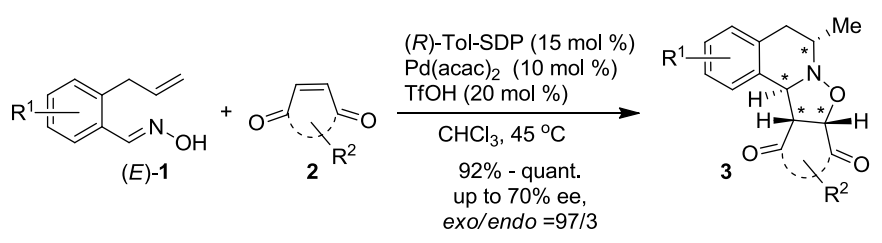

Scheme 1 Pd(II)-catalyzed diastereoselective and enantioselective domino cyclization/cycloaddition reaction of alkenyl oxime $\mathbf{1}$ and enedione 2 .
With the aim of developing a diastereoselective and enantioselective cyclization/cycloaddition sequence, the reaction of alkenyl oxime $(E)$-1a and $N$-methyl maleimide (2a) as prototypical substrates was attempted (Table 1). ${ }^{4}$ Although a chiral complex derived from $\mathrm{PdCl}_{2}(\mathrm{MeCN})_{2}$ with $(S)$-BINAP was used, no desired cyclic product 3a was obtained. ESI-MS studies of the reaction indicated the formation of intermediate $4 .^{4,6}$ To promote the protonolysis of the $\mathrm{Pd}-\mathrm{C}$ bond in $\mathbf{4}$ that lead to the domino process, various $\mathrm{Br} \phi$ nsted acids as proton sources were employed. Among the acids tested $\left(\mathrm{HClO}_{4}, \mathrm{HCl}_{\mathrm{aq}}, p\right.$-Tol- $\mathrm{SO}_{3} \mathrm{H}$, $\mathrm{CF}_{3} \mathrm{CO}_{2} \mathrm{H}, o-\mathrm{NO}_{2}-\mathrm{C}_{6} \mathrm{H}_{4}-\mathrm{CO}_{2} \mathrm{H}, \mathrm{HCO}_{2} \mathrm{H}$, and $\mathrm{AcOH}$ ), the addition of $\mathrm{TfOH}$ was found to promote the domino process effectively, resulting in the formation of $\mathbf{3 a}$ with $70 \%$ yields in the ratio of exo/endo-3a to 93/7; the exo-cycloadduct 3a was obtained as a major product in $37 \%$ enantiomeric excess (ee) (Entry 2). Encouraged by these results, we further studied the effects of other reaction conditions such as solvents, Pd salts, a ratio of substrates, and temperature. The use of $\mathrm{CHCl}_{3}$ as a reaction solvent resulted in the formation of $\mathbf{3 a}$ with $46 \%$ ee (Entry 4$)$. Chiral Pd complexes prepared from $\mathrm{Pd}(\mathrm{acac})_{2}$ with $(S)$-BINAP were found to give 3a in higher enantioselectivity (55\% ee, Entry 7) than those prepared from other Pd salts. The optimal result (quant, exo/endo $=93 / 7,56 \%$ ee) with $\mathrm{Pd}(\mathrm{II})-(S)$-BINAP was obtained when the reaction of $\mathbf{1 a}$ with $\mathbf{2 a}$ in a ratio of 2 to 1 was performed in $\mathrm{CHCl}_{3}$ at $45^{\circ} \mathrm{C}$ (Entry 10).

Next, we focused on searching for an appropriate chiral ligand to construct isoxazolidine 3a with high optical purity (Table 2). The catalysts derived from $\mathrm{Pd}(\mathrm{acac})_{2}$ with $(S)$-Tol-BINAP, $(R)-C_{3^{-}}$ Tunephos, or $(R)$-Segphos were virtually ineffective in improving

* Corresponding author. Tel.: +81-6-6879-8466; fax: +81-6-6879-8469; e-mail: taki@ sanken.osaka-u.ac.jp 
Table 1 Screening of reaction conditions. ${ }^{a}$

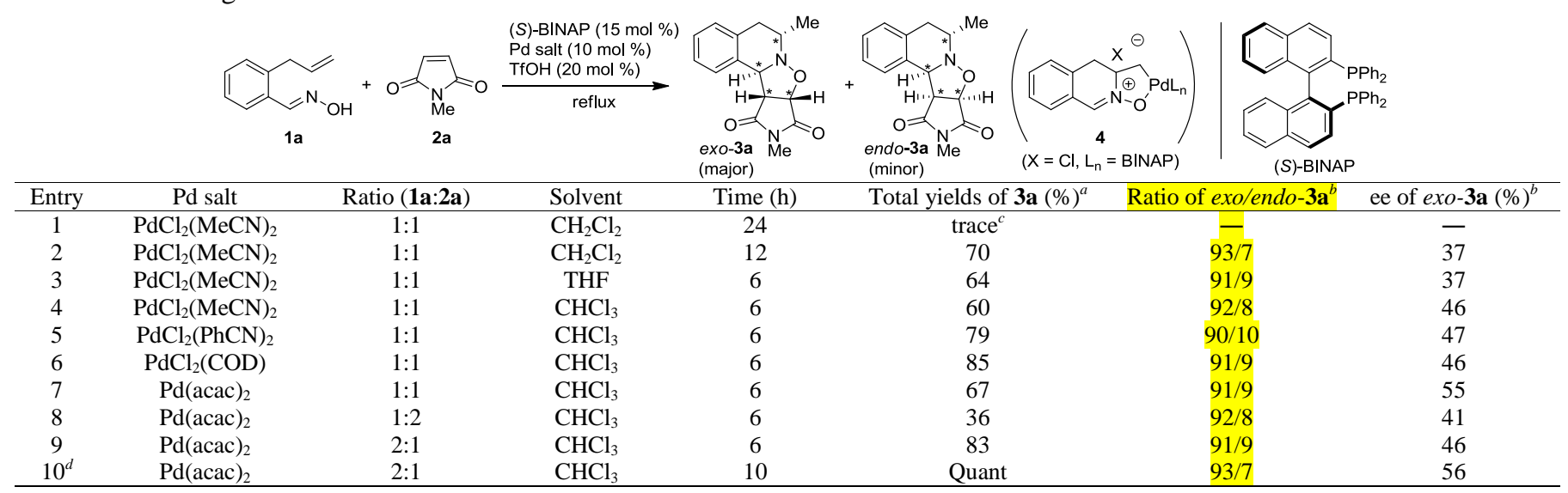

${ }^{a}$ Isolated yield.

${ }^{b}$ Determined by HPLC.

${ }^{c}$ In the absence of $\mathrm{TfOH}$

${ }^{d}$ At $45^{\circ} \mathrm{C}$

the stereoselectivity (Entries 1-3). Because a larger bite angle of the chiral ligand is suggested to increase the ee value of domino product $3 \mathbf{a}^{7}$ we shifted our attention to utilizing a chiral spirotype ligand having a wide bite angle. A spiro-type ligand would provide a geometrically distinct and more rigid chiral pocket than its BINOL-derived counterpart. ${ }^{8}$ As expected, the $\mathrm{Pd}(\mathrm{acac})_{2} /(R)$ SDP system was found to be efficient in affording good enantioselectivity (62\% ee, Entry 4$)$. An ee of $70 \%$ was achieved with the $\operatorname{Pd}(\mathrm{acac})_{2} /(R)$-Tol-SDP catalyst (Entry 5). The use of

Table 2 Screening of chiral ligands. ${ }^{a}$

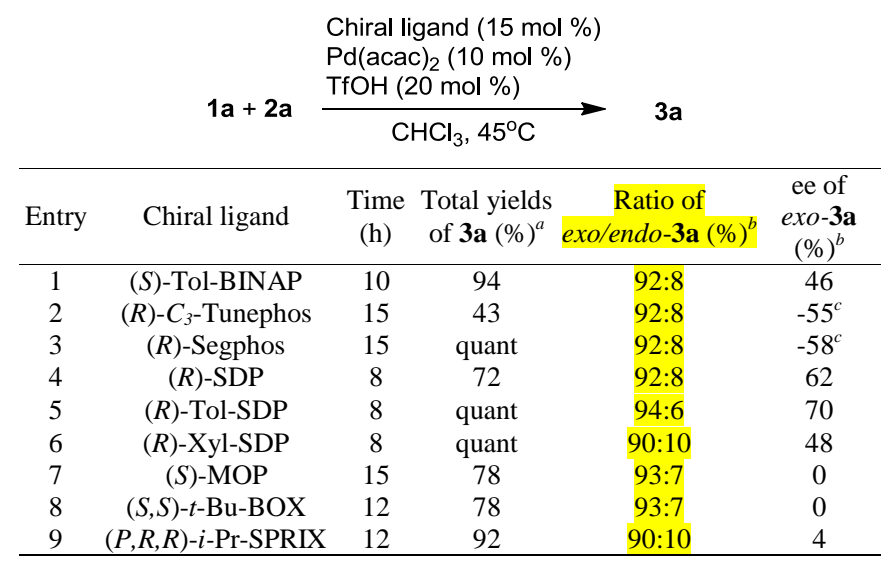

${ }^{a}$ Isolated yield.

${ }^{b}$ determined by HPLC.

${ }^{c}$ Opposite enantiomer was obtained.
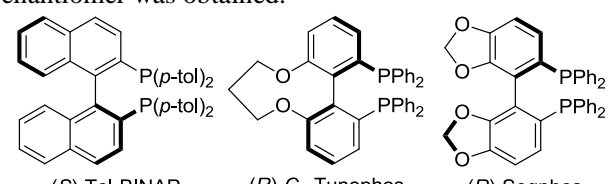

(S)-Tol-BINAP

$(R)-C_{3}$-Tunephos

(R)-Segphos
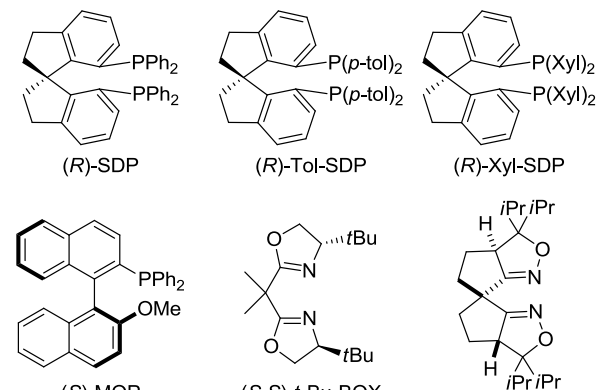

(S)-MOP<smiles>CC(C)OC(C(C)C)C1CCCC12CCCC1C2=NOC1(C(C)C)C(C)C</smiles>

$(P, R, R)$-iPr-SPRIX

(R)-Xyl-SDP possessing bulky substituents on the phosphine region resulted in diminished enantioselectivity (Entry 6). Pd(II) complexes of other chiral monodentate phosphine ligands, $(S)$ MOP, and chiral bidentate nitrogen ligands, such as $(S, S)-t$ BuBOX or $(P, R, R)-i$-Pr-SPRIX ${ }^{8 \mathrm{a}, 9}$ failed to induce enantioselectivity (Entries 7-9). The relatively weakly coordinated ligands might be replaced by the substrate, allowing the background reaction to afford the racemic product. ${ }^{8 \mathrm{~d}}$ In fact, after the addition of $(E)$-1a to the chiral Pd(II) complex derived from SPRIX, only the dissociated free ligand signals were observed in the ${ }^{1} \mathrm{H}-\mathrm{NMR}$ spectra.
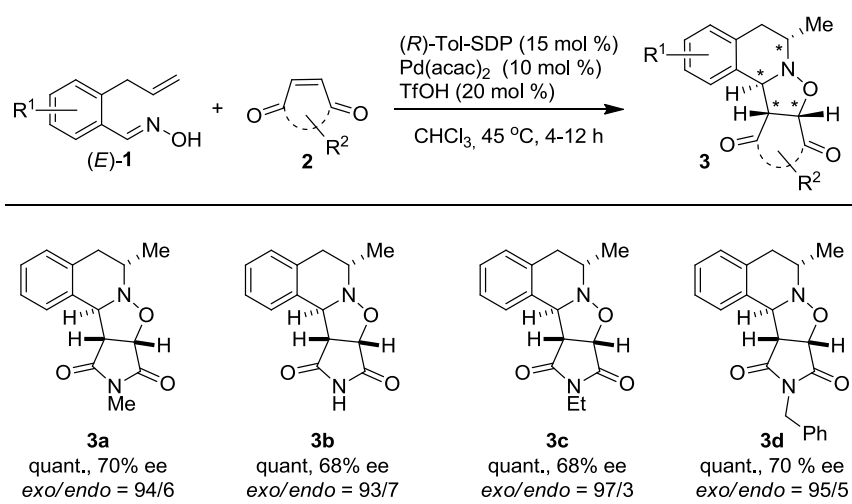

$$
3 \mathrm{c}
$$

quant., $68 \%$ ee

exo/endo $=97 / 3$
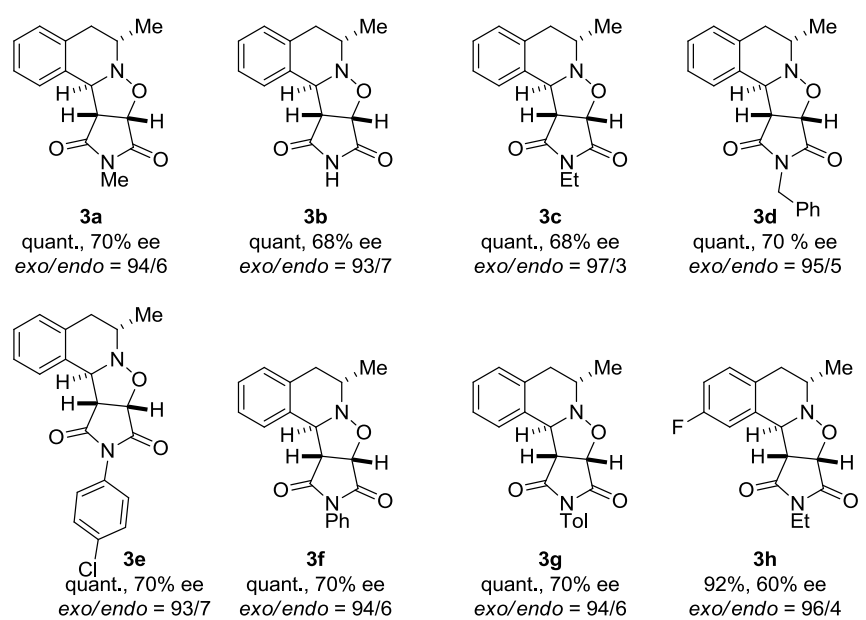

$3 \mathbf{g}$

quant., $70 \%$ ee

exo/endo $=94 / 6$

3h

$92 \%, 60 \%$ ee

exo/endo $=96 / 4$
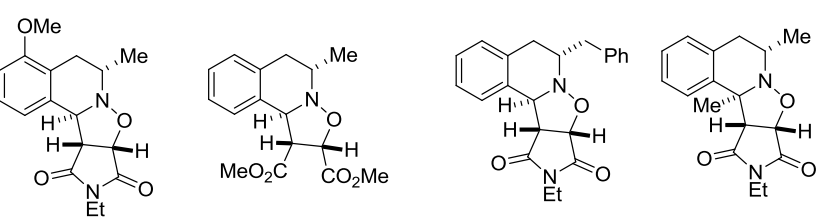

3k

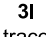

trace

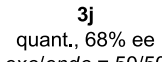
exo/endo $=50 / 50$

trace 
The scope and limitations of this protocol were then studied by employing the optimized reaction conditions $\left(\operatorname{Pd}(\mathrm{acac})_{2} /(R)\right.$-Tol$\left.\mathrm{SDP}, 45{ }^{\circ} \mathrm{C}, \mathrm{CHCl}_{3}\right)$ to the enantioselective domino cyclization (Scheme 2). The reaction of alkenyl oxime $(E)-\mathbf{1 a}$ and maleimides 2 bearing various substituents on the nitrogen atom afforded the corresponding tetracycles $\mathbf{3 a}-\mathbf{3 g}$ with four chiral stereogenic centers in quantitative yields with good stereoselectivities $(68 \%-70 \%$ ee, exo/endo $=93 / 7-97 / 3)$. Alkenyl oximes $(E)$-1h and $(E)$-1i bearing an electron-withdrawing or electron-donating substituent on the aromatic rings also afforded domino products $\mathbf{3 h}$ and $\mathbf{3 i}$ in excellent yields with moderate enantioselectivities. When using $(E)$-1a and dimethyl maleate for the domino reaction, the corresponding exo- and endocycloadduct $\mathbf{3 j}$ in a ratio of $1: 1$ were obtained in $68 \%$ ees, respectively. No desired product was formed when using $\mathbf{1 k}$, having a phenyl substituent at the terminal olefin moiety, and ketoxime $11,{ }^{10}$ presumably because of the bulky substituents preventing catalyst activation.

A plausible reaction mechanism for the $\mathrm{Pd}(\mathrm{II})$-catalyzed enantioselective domino cyclization/cycloaddition reactions is shown in Figure 1. The initial coordination of the chiral Pd(II)$(R)$-Tol-SDP complex with alkenyl oxime 1 led to transition state I. The subsequent nucleopalladation of $\mathbf{I}$ afforded the cyclic intermediate II and III, thus controlling the enantioselectivity. The protonation of generated Pd(II) intermediate II (or III) made the releasing catalyst, together with the formation of nitrone IV. Finally, 1,3-dipolar cycloaddition of IV with $\mathbf{2}$ gave domino product 3 .

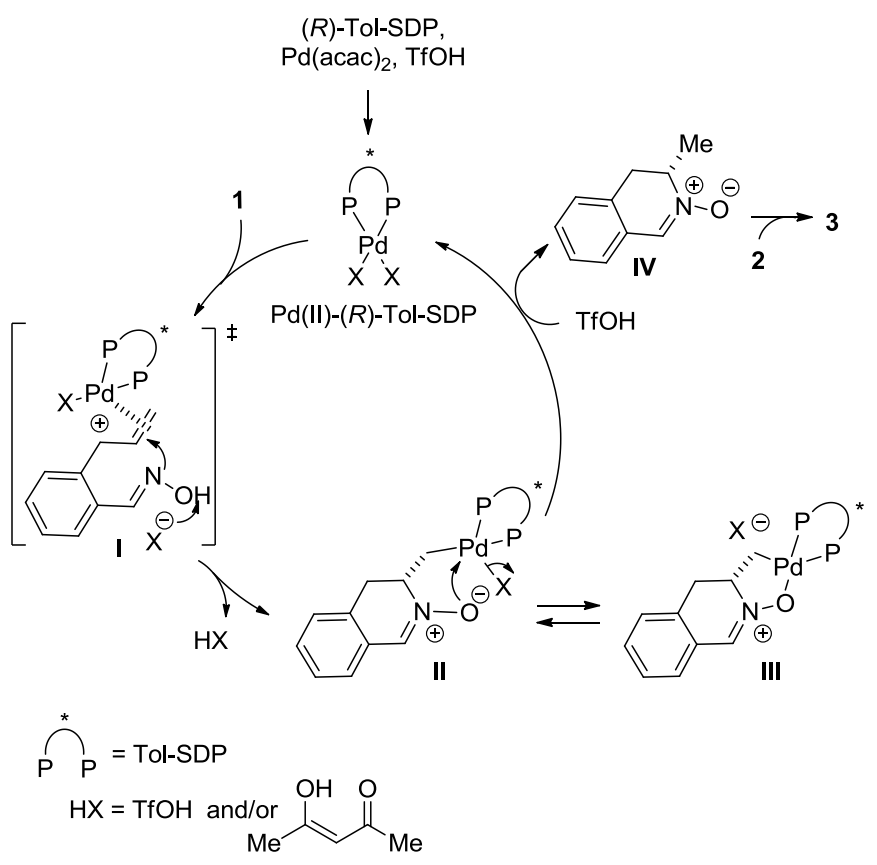

Figure 1 Plausible reaction mechanism.

In summary, we have developed a domino cyclization/cycloaddition of alkenyl oximes catalyzed by the chiral $\mathrm{Pd}(\mathrm{II})$ complex and $\mathrm{TfOH}$. The use of the $(R)$-TolSDP ligand led to the formation of polycyclic heterocycles having four chiral stereogenic centers in almost quantitative yields with high stereoselectivities. A fine-tuning of the spiro catalysts, investigation into the detailed reaction mechanism, scope, as well as its application to the enantioselective synthesis of biologically active compounds are currently underway.

\section{Acknowledgments}

This study was supported by a Grant-in-Aid for Scientific Research on Innovative Areas -Advanced Molecular Transformations by Organocatalysis- from The Ministry of Education, Culture, Sports, Science and Technology (MEXT), Japan, the CREST project of the Japan Science and Technology Corporation (JST), and JST Advance Catalytic Transformation Program for Carbon Utilization (ACT-C). We acknowledge the technical staff of the Comprehensive Analysis Center of ISIR, Osaka University (Japan).

\section{References and notes}

1. For selected reviews, see: (a) Tietze, L. F. Modi, A. Med. Res. Rev 2000, 20, 304; (b) Kitamura, M. Narasaka, K. Chem. Rec. 2002, 2, 268; (c) Zeni, G. Larock, R. C. Chem. Rev. 2004, 104, 2285; (d) Minatti, A. Muniz, K. Chem. Soc. Rev. 2007, 36, 1142; (e) Tao, H.-Y. Wang, C.-J. Synlett, 2014, 25, 461; (f) Najera, C. Sansano, J. M. J. Organomet. Chem. 2014, 771, 78; (g) Narayan, R.; Potowski, M. Jia, Z.-J. Antonchick, A. P. Waldmann, H. Acc. Chem. Res. 2014, 47, 1296; (h) Adrio, J. Carretero, J. C. Chem. Commun. 2014, 50, 12434 .

2. For selected reviews on domino process for natural product synthesis, see: (a) Tietze, L. F. J. Heterocyclic Chem. 1990, 27, 47; (b) Overman, L. E. Pennington, L. D. J. Org. Chem. 2003, 68, 7143; (c) Chapman, C. J. Frost, C. G. Synthesis 2007, 1; (d) Padwa, A. Chem. Soc. Rev. 2009, 38, 3072; (e) Grondal, C. Jeanty, M. Enders, D. Nature Chem. 2010, 2, 167; (f) In Domino Reactions: Concepts for Efficient Organic Synthesis (Tietze, L. F. Ed) Wiley-VCH Verlag GmbH \& Co. KGaA (2014).

3. (a) Grigg, R. Markandu, J. Perrior, T. Surendrakumar, S. Warnock, W. J. Tetrahedron Lett. 1990, 31, 559; (b) Armstrong, P. Grigg, R. Heaney, F. Surendrkumar, S. Warnock, W. J. Tetrahedron 1991, 47, 4495; (c) Grigg, R. Hadjisoteriou, M. Kennewell, P. Markandu J. Thornton-Pett, M. J. Chem. Soc., Chem. Commun. 1992, 1388; (d) Grigg, R. Hadjisoteriou, M. Kennewell, P. Markandu, J. J. Chem. Soc., Chem. Commun. 1992, 1537; (e) Grigg, R. Hadjisoteriou, M. Kennewell, P. Markandu, J. Thornton-Pett, M. J. Chem. Soc., Chem. Commun. 1993, 1340; (f) Frederickson, M. Grigg, R. Thornton-Pett, M. Redpath, J. Tetrahedron Lett. 1997, 38, 7777; (g) Greci, L. Tommasi, G. Bruni, P. Sgarabotto, P. Righi, L. Eur. J. Org. Chem. 2001, 3147; (h) Horsley, H. T. Holmes, A. B. Davies, J. E. Goodman, J. M. Silva, M. A. Pascu, S. I. Collins, I. Org. Biomol. Chem. 2004, 2, 1258; (i) Gucma, M. Golebiewski, W. M. J. Heterocyclic Chem. 2008, 45, 241; (j) Burrell, A. J. M. Coldham, I. Oram, N. Org. Lett. 2009, 11, 1515; (k) Burrell, A. J. M. Coldham, I. Watson, L. Oram, N. Pilgram, C. D. Martin, N. G. J. Org. Chem. 2009, 74, 2290; (1) Zhang, Y. Stephens, D. Hernandez, G. Mendoza, R. Larionov, O. V. Chem. Eur. J. 2012, 18, 16612; (m) Buchlovic, M. Hebanova, S. Potacek, M. Tetrahedron 2012, 68, 3117; (n) Mehra, V. Kumar, V. Tetrahedron Lett. 2014, 55, 845.

4. (a) Frederickson, M. Grigg, R. Markandu, J. Redpath, J. J. Chem. Soc., Chem. Commun. 1994, 2225; (b) Frederickson, M. Grigg, R. Markandu, J. Thornton-Pett, M. Redpath, J. Tetrahedron 1997, 53, 15051 .

5. The asymmetric domino process using a chiral auxiliary was reported, see: (a) Williams, G. M. Roughley, S. D. Davies, J. E. Holmes, A. B. J. Am. Chem. Soc. 1999, 121, 4900; (b) Tamura, O. Mita, N. Okabe, T. Yamaguchi, T. Fukushima, C. Yamashita, M. Morita, Y. Morita, N. Ishibashi, H. Sakamoto, M. J. Org. Chem. 2001, 66, 2602; (c) Osborn, H. M. I. Gemmell, N. Harwood, L. M. J. Chem. Soc., Perkin Trans. 1 2002, 2419; (d) Davison, E. C. Fox, M. E. Holmes, A. B. Roughley, S. D. Smith, C. J. Williams, G. M. Davies, J. E. Raithby, P. R. Adams, J. P. Forbes, I. T. Press, N. J. Thompson, M. J. J. Chem. Soc., Perkin Trans. 1 2002, 1494.

6. Formation of intermediate $\mathbf{4}$ was supported by comparison an isotope pattern of $\mathbf{4}$ with the calculated value on ESI-MS; intermediate 4: m/z: $888(100 \%), 890(75 \%), 887(68 \%), 889$ (48\%), 891 (39\%), 892 (38\%), 886 (26\%), 893 (18\%).

7. (a) Dierkes, P. van Leeuwen, P. W. N. M. J. Chem Soc., Dalton Trans. 1999, 1519; (b) Freixa, Z. van Leeuwen, P. W. N. M. Coord. Chem. Rev. 2008, 252, 1755; (c) Czauderna, C. F. Cordes, D. B. Slawin, A. M. Z. Müller, C. van der Vlugt, J. I. Vogt, D. Kamer, P. C. J. Eur. J. Inorg. Chem. 2014, 1797.

8. (a) Bajracharya, G. B. Arai, M. A. Koranne, P. S. Suzuki, T. Takizawa, S. Sasai, H. Bull. Chem. Soc. Jpn. 2009, 82, 285; (b) Ding, K. Han, Z. Wang, Z. Chem. Asian J. 2009, 4, 32; (c) Zhu, 
Tetrahedron

S.-F. Zhou, Q.-L. Acc. Chem. Res. 2012, 45, 1365; (d) Sridharan, V. Fan, L. Takizawa, S. Suzuki, T. Sasai, H. Org. Biomol. Chem. 2013, 11, 5936.

9. (a) Tsujihara, T. Takenaka, K. Onitsuka, K. Hatanaka, M. Sasai, H J. Am. Chem. Soc. 2009, 131, 3452; (b) Gabr, R. K. Hatakeyama, T. Takenaka, K. Takizawa, S. Okada, Y. Nakamura, M. Sasai, H. Chem. Eur. J. 2013, 19, 9518; (c) Takenaka, K. Mohanta, S. C. Sasai, H. Angew. Chem. Int. Ed. 2014, 53, 4675.

10. Although the corresponding intermediate IV was formed when using ketoxime 11, no cycloaddition with $N$-ethyl maleimide (2c) was occurred

11. Yields of isolated 3. The ee and ratio of exolendo-3 were determined by HPLC $。$ Determination of absolute configuration of $\mathbf{3}$ is in progress. 
+Supporting Information

\title{
Pd(II)-catalyzed diastereoselective and enantioselective domino cyclization/cycloaddition reactions of alkenyl oximes for polycyclic heterocycles with four chiral stereogenic centers
}

\author{
Mohamed A. Abozeid, Shinobu Takizawa, ${ }^{*}$ and Hiroaki Sasai \\ The Institute of Scientific and Industrial Research (ISIR), Osaka University \\ Mihogaoka, Ibaraki-shi, Osaka 567-0047, Japan. \\ E-mail: taki@sanken.osaka-u.ac.jp
}

\section{Contents:}

A. General information (P-2)

B. Preparation of starting material $1 \quad$ (P-2)

C. Ligand screening with $\mathrm{Pd}\left(\mathrm{F}_{6} \text {-acac }\right)_{2}$ at $40{ }^{\circ} \mathrm{C} \quad$ (P-3)

D. General procedure for Pd(II)-catalyzed diastereoselective and enantioselective domino cyclization/cycloaddition reactions of alkenyl oximes $\quad(P-5)$

E. References (P-9)

F. NMR charts (P-10)

G. HPLC charts (P-20) 


\section{A. General information}

All reactions were performed with standard Schlenk technique under $\mathrm{N}_{2}$ atmosphere. ${ }^{1} \mathrm{H}-,{ }^{13} \mathrm{C}$ - and ${ }^{19} \mathrm{~F}-\mathrm{NMR}$ spectra were recorded with JEOL JMN LA-400 FT NMR or JNM ECA600 FT NMR ( ${ }^{1} \mathrm{H}-\mathrm{NMR} 600$ or $400 \mathrm{MHz}$, ${ }^{13} \mathrm{C}$-NMR 150 or $100 \mathrm{MHz}$ ). ${ }^{1} \mathrm{H}-\mathrm{NMR}$ spectra are reported in ppm $(\delta)$ relative to the chemical shift of tetramethylsilane. Multiplicity ( $\mathrm{s}=$ singlet, $\mathrm{d}=$ doublet, $\mathrm{q}=$ quartet, $\mathrm{t}=$ triplet, $\mathrm{m}=$ multiplet) ${ }^{13} \mathrm{C}-\mathrm{NMR}$ spectra reported in ppm $(\delta)$ relative to the central line of triplet for $\mathrm{CDCl}_{3}$ at $77.16 \mathrm{ppm} . \mathrm{CF}_{3} \mathrm{CO}_{2} \mathrm{H}$ used as external standards for ${ }^{19} \mathrm{~F}-\mathrm{NMR}$. ESI-MS spectra were obtained with JMS-T100LC (JEOL). Optical rotations were measured with JASCO P-1030 polarimeter. HPLC analyses were performed on a JASCO HPLC system (JASCO PU 980 pump and UV-975 UV/Vis detector) using a mixture of hexane and $i$-PrOH eluents. FT-IR spectra were recorded on a JASCO FT-IR system (FT/IR4100). Melting point was measured with SHIMADZU DSC-60. Column chromatography on $\mathrm{SiO}_{2}$ was performed with Kanto Silica Gel $60(40-100 \mu \mathrm{m})$. Commercially available organic and inorganic compounds were used without further purification. The solvents were purified and dried by the standard procedures. 2-Aallyl-5-fluorobenzaldehyde (S1b) ${ }^{1}, 2$-allyl-3-methoxybenzaldehyde (S1c), ${ }^{2}$ and $(E)-\mathbf{1} \mathbf{a}^{3}$ were synthesized according to the reported procedures. Domino products $\mathbf{3 a - j}$ are inseparable diastereomeric mixtures. In the case of minor diastereomer, only the representative peaks were exhibited in the ${ }^{1} \mathrm{H}$-NMR data. The relative stereochemistry of the domino products 3a-3i was assigned based on Grigg's analogy. ${ }^{3}$ The relative stereochemistry of domino product $\mathbf{3 j}$ was assigned using gradient $1 \mathrm{D}-\mathrm{NOE}$ studies. The indicated $[\alpha]_{D}^{T}$ value for the domino products 3a-i were measured for the diastereomeric mixture (inseparable) except for the domino product $\mathbf{3} \mathbf{j}$ in which the two diastereomers are separable.

\section{B. Preparation of starting material 1}

To a mixture of aldehyde $(1.37 \mathrm{mmol})$ and pyridine $(165 \mu \mathrm{L}, 2.05 \mathrm{mmol}, 1.5 \mathrm{eq}$.$) in methanol (2 \mathrm{~mL})$, a solution of hydroxylamine hydrochloride $(95.2 \mathrm{mg}, 1.37 \mathrm{mmol})$ in water $(0.28 \mathrm{~mL})$ was added and the mixture was stirred at room temperature overnight. The solvent was removed in vacuo and the residue was treated with $2 \mathrm{M}$ $\mathrm{HCl}(8.2 \mathrm{ml})$. The organic material was extracted into diethyl ether $(3 \times 10 \mathrm{~mL})$. The combined organic layers were dried over anhydrous sodium sulphate and the solvent was removed in vacuo. The remaining residue was further purified by column chromatography on silica (Hexane : $\left.\mathrm{Et}_{2} \mathrm{O}=95: 5\right)$ to give the pure alkenyl oximes 1b-c.

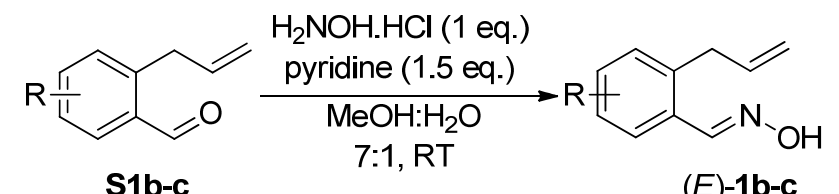

S1b-c

$(E)-1 \mathbf{b}-\mathbf{c}$<smiles>C=CCc1ccc(F)cc1/C=N/O</smiles>

1b<smiles>C=CCc1c(/C=N/O)cccc1OC</smiles> 


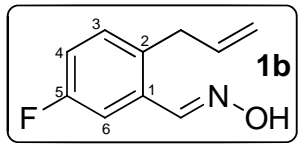

1b: colorless oil (79\%); ${ }^{1} \mathrm{H}-\mathrm{NMR}\left(\mathrm{CDCl}_{3}\right) \delta 8.33(\mathrm{~d}, 1 \mathrm{H}, \underline{\mathrm{CH}}=\mathrm{NOH}, J=1.60 \mathrm{~Hz}), 7.66$ $(\mathrm{s}, 1 \mathrm{H}, \mathrm{OH}), 7.45(\mathrm{dd}, 1 \mathrm{H}, \mathrm{H}-6, J=2.80,9.60 \mathrm{~Hz}), 7.16(\mathrm{dd}, 1 \mathrm{H}, \mathrm{H}-3, J=5.20,8.00 \mathrm{~Hz})$, $7.03(\mathrm{td}, 1 \mathrm{H}, \mathrm{H}-4, J=2.80,8.00,10.80 \mathrm{~Hz}), 6.00-5.90\left(\mathrm{~m}, 1 \mathrm{H}, \underline{\mathrm{CH}}=\mathrm{CH}_{2}\right), 5.10(\mathrm{dd}, 1 \mathrm{H}$, $\left.\underline{\mathrm{CH}}=\mathrm{CH}_{2 \mathrm{a}}, J=1.20,10.00 \mathrm{~Hz}\right), 4.94\left(\mathrm{dd}, 1 \mathrm{H}, \mathrm{CH}=\underline{\mathrm{CH}}_{2 \mathrm{~b}}, J=1.20,18.80 \mathrm{~Hz}\right), 3.47\left(\mathrm{~d}, 2 \mathrm{H}, \underline{\mathrm{CH}_{2}} \mathrm{CH} \mathrm{CH}_{2}, J=\right.$ $6.00 \mathrm{~Hz}) ;{ }^{13} \mathrm{C}-\mathrm{NMR}\left(\mathrm{CDCl}_{3}\right) \delta 148.1,136.6,132.1,132.0,117.2,117.0,116.7,113.0,112.9,36.5 ;{ }^{19} \mathrm{~F}-\mathrm{NMR}$ $\left(\mathrm{CDCl}_{3}\right): \delta$-115.8; HRMS (ESI) calcd for $\mathrm{C}_{10} \mathrm{H}_{10} \mathrm{FNNaO}, \mathrm{m} / \mathrm{z}=202.0639\left[(\mathrm{M}+\mathrm{Na})^{+}\right]$, found $\mathrm{m} / \mathrm{z}=202.0637$; IR $(\mathrm{KBr}): v 3357,3082,3006,2980,2923,1638,1609,1580,1496,1421 \mathrm{~cm}^{-1}$.

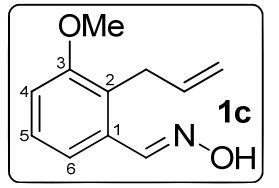

1c: yellow solid $(81 \%) ; 92-93{ }^{\circ} \mathrm{C} ;{ }^{1} \mathrm{H}-\mathrm{NMR}\left(\mathrm{CDCl}_{3}\right) \delta 8.63(\mathrm{~s}, 1 \mathrm{H}, \mathrm{OH}), 8.4(\mathrm{~s}, 1 \mathrm{H}$, $\underline{\mathrm{CH}}=\mathrm{NOH}), 7.35(\mathrm{~d}, 1 \mathrm{H}, \mathrm{H}-6, J=7.60 \mathrm{~Hz}), 7.22(\mathrm{dd}, 1 \mathrm{H}, \mathrm{H}-5, J=7.60,8.40 \mathrm{~Hz}), 6.91(\mathrm{~d}$, $1 \mathrm{H}, \mathrm{H}-4, J=8.40 \mathrm{~Hz}), 5.99-5.89\left(\mathrm{~m}, 1 \mathrm{H}, \underline{\mathrm{CH}}=\mathrm{CH}_{2}\right), 5.00\left(\mathrm{dd}, 1 \mathrm{H}, \mathrm{CH}=\underline{\mathrm{CH}}_{2 \mathrm{a}}, J=1.60\right.$, $10.00 \mathrm{~Hz}), 4.89\left(\mathrm{dd}, 1 \mathrm{H}, \mathrm{CH}=\underline{\mathrm{CH}}_{2} \mathrm{~b}, J=1.60,15.60 \mathrm{~Hz}\right), 3.54\left(\mathrm{~s}, 3 \mathrm{H}, \mathrm{OCH}_{3}\right), 3.54(\mathrm{~d}, 2 \mathrm{H}$, $\left.\underline{\mathrm{CH}_{2}} \mathrm{CH}=\mathrm{CH}_{2}, J=5.60 \mathrm{~Hz}\right) ;{ }^{13} \mathrm{C}-\mathrm{NMR}\left(\mathrm{CDCl}_{3}\right) \delta 157.7,149.0,136.0,131.7,127.5,127.4,118.7,115.4,112.0$, 55.9, 29.4; HRMS (ESI) calcd for $\mathrm{C}_{11} \mathrm{H}_{13} \mathrm{NNaO}_{2}, \mathrm{~m} / \mathrm{z}=214.0838\left[(\mathrm{M}+\mathrm{Na})^{+}\right]$, found $\mathrm{m} / \mathrm{z}=214.0838$; IR (KBr): $v 3345,3081,3006,3918,2838,1634,1578,1470,1449,1437,1406 \mathrm{~cm}^{-1}$.

\section{Ligand screening with $\operatorname{Pd}\left(\mathrm{F}_{6} \text {-acac }\right)_{2}$ at $40{ }^{\circ} \mathrm{C}$}

\section{Table S1}
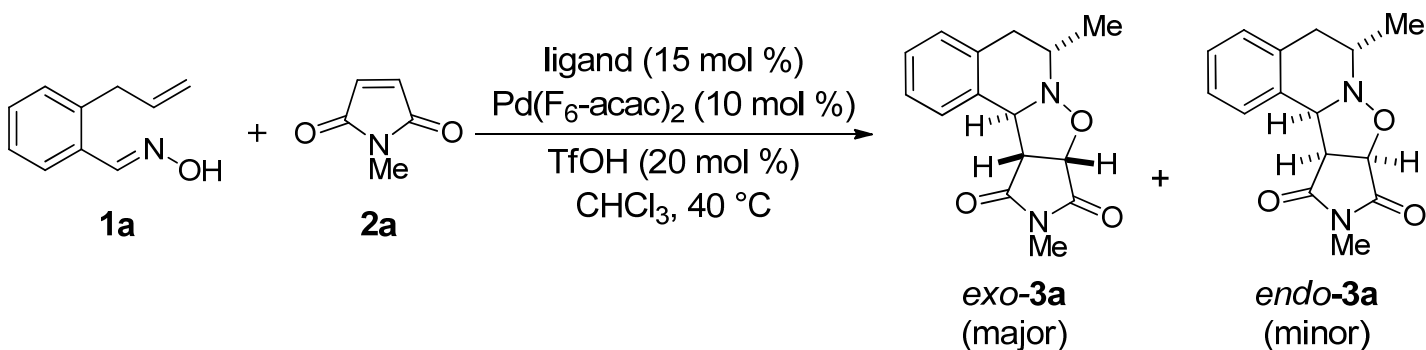

endo-3a (minor)

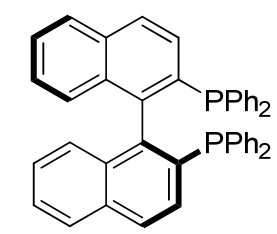

(S)-BINAP (18h) $71 \%, 45 \%$ ee exo/endo $=94 / 6$

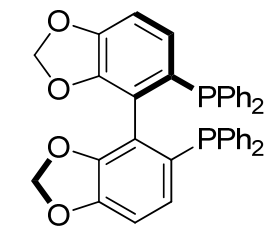

(R)-Segphos (12h) $58 \%,-52 \%$ ee exo $/$ endo $=94 / 6$

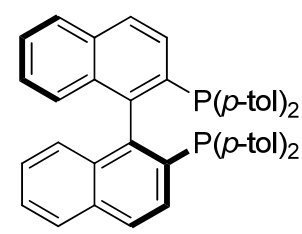

(S)-Tol-BINAP (18h) $65 \%, 37 \%$ ee exo/endo $=94 / 6$<smiles>Cc1cc(C)cc(Pc2ccc3c(c2-c2c(Pc4cc(C)cc(C)c4)ccc4c2OCO4)OCO3)c1</smiles>

(R)-DM-Segphos (12h)

$56 \%,-45 \%$ ee exo/endo $=94 / 6$

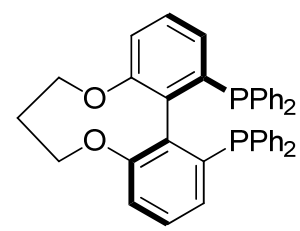

(R)- $\boldsymbol{C}_{3}$-Tunephos $75 \%,-48 \%$ ee exo $/$ endo $=94 / 6$<smiles>FC1(F)Oc2ccc(P)c(-c3c(P)ccc4c3OC(F)(F)O4)c2O1</smiles>

(R)-Difluorophos (24h) $72 \%,-45 \%$ ee exo $/$ endo $=94 / 6$

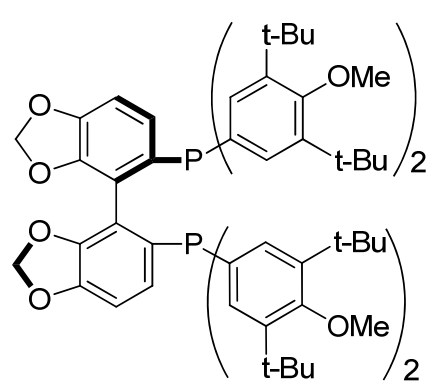

(R)-DTBM-Segphos (24h) $52 \%, 35 \%$ ee exo/endo $=97 / 3$ 
Table S1 (continue)<smiles>[Y10]P(c1nc2ccccc2nc1P(C)C)C(C)(C)C</smiles>

$(\boldsymbol{R}, \boldsymbol{R})$-QuinoxP* (12h) $80 \%,-24 \%$ ee exo/endo $=95 / 5$<smiles>c1ccc(C2CC[C@H](c3ccccc3)P2CCP2[C@H](c3ccccc3)CC[C@@H]2c2ccccc2)cc1</smiles>

$(\boldsymbol{R}, \boldsymbol{R})$-Ph-BPE (12h) $80 \%, 52 \%$ ee exo/endo $=95 / 5$<smiles>CCCCP(C)c1ccccc1P(C)C(C)(C)C</smiles>

$(S, S)-B e n z P *(12 h)$ $80 \%, 31 \%$ ee exo/endo $=94 / 6$<smiles>CC(C)(C)P1Cc2ccccc2C1C1c2ccccc2C[PH]1C(C)(C)C</smiles>

(R,R,S,S)-Duanphos (12h)

$79 \%, r a c$. exo/endo $=94 / 6$<smiles>CCCC1CCC(P2CCC(C(C)C)P2c2ccccc2P(C)C)C1</smiles>

'Pr-Duphos (12h)

$63 \%, 8 \%$ ee

exo/endo $=94 / 6$<smiles>CC(P)C(C)P(c1ccccc1)c1ccccc1</smiles>

$(\boldsymbol{R}, \boldsymbol{R})$-Chiraphos (8h)

$69 \%,-7 \%$ ee exo/endo $=94 / 6$<smiles>Pc1ccccc1</smiles>

(S,S)-Norphos (12h) $58 \%,-5 \%$ ee exo/endo $=96 / 4$<smiles>CC1(CO)CCC(CPc2ccccc2)C1(C)CPc1ccccc1</smiles>

[(1R,2R,3S)-(+)-1,2-Dimethyl-2,3bis(diphenylphosphinomethyl)cy clopentyl]methanol (48h)

$74 \%, 34 \%$ ee exo/endo $=97 / 3$<smiles>CC1(C)OC(CPc2ccccc2)[C@H](Cc2ccccc2)O1</smiles>

(R,R)-DIOP (12h) $72 \%$, rac. exo/endo $=94 / 6$

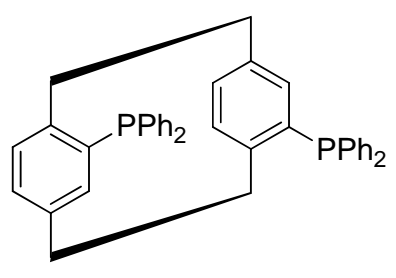

(R)-Phanephos (5 days) $55 \%, 18 \%$ ee exo/endo $=94 / 6$<smiles></smiles>

$(\boldsymbol{R}, \boldsymbol{R})$-Chiraphite (36h) $55 \%$, rac exo/endo $=95 / 5$<smiles>CC(=O)Oc1ccccc1-c1ccccc1Pc1ccccc1</smiles>

$(R, S)-B P P F O A C ~(24 h)$ $46 \%,-17 \%$ ee exo/endo $=94 / 6$<smiles>CN(C)p1oc2ccc3ccccc3c2c2c(ccc3ccccc32)o1</smiles>

(S)-Monophos (12h)

$61 \%,-7 \%$ ee exo/endo $=96 / 4$<smiles>CC(c1ccc(Pc2ccccc2)c(Pc2ccccc2)c1)C(C(C)(C)C)C(C)(C)C</smiles>

Josiphos (J002-2) (48h)

$15 \%$ (Con. $=71 \%$ ), $9 \%$ ee exo/endo $=95 / 5$

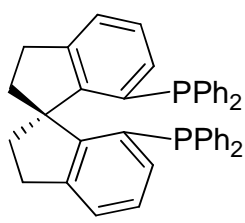

(R)-SDP (18h)

$68 \%, 68 \%$ ee exo/endo $=94 / 6$<smiles>Pc1cccc(Nc2ccc3ccccc3c2-c2c(NPc3ccccc3)ccc3ccccc23)c1P</smiles>

(R)-BINAM-P (24h) $83 \%, r a c$. exo/endo $=93 / 7$

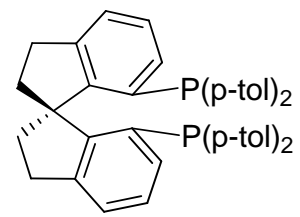

(R)-Tol-SDP

At $40{ }^{\circ} \mathrm{C}$ (12h): $63 \%$, $73 \%$ ee exo/endo $=94 / 6$

At $45{ }^{\circ} \mathrm{C}$ (8h): $69 \%$, $74 \%$ ee exo/endo $=94 / 6$<smiles>Pc1cccc(-c2nccc3ccccc23)c1-c1nccc2ccccc12</smiles>

(R)-QUINAP (4days) No reaction

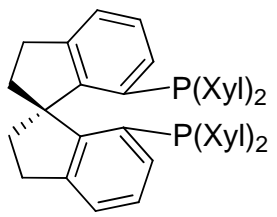

(R)-Xyl-SDP (12h) $64 \%, 68 \%$ ee exo/endo $=94 / 6$ 


\section{General procedure for Pd(II)-catalyzed diastereoselective and enantioselective domino}

cyclization/cycloaddition reactions of alkenyl oximes

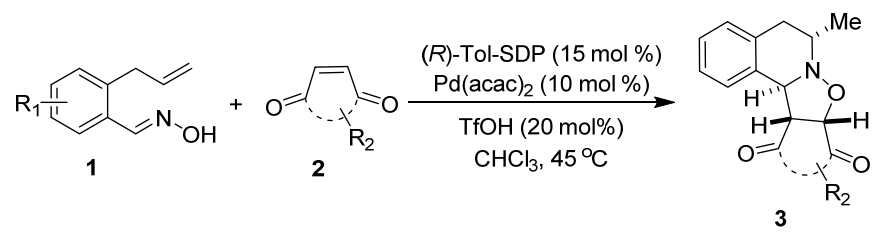

In $10 \mathrm{ml} \mathrm{R.B.} \mathrm{flask,} \mathrm{a} \mathrm{mixture} \mathrm{of} \mathrm{(R)-Tol-SDP} \mathrm{(6} \mathrm{mg,} 0.0093 \mathrm{mmol}, 15 \mathrm{~mol} \%)$ and Pd(acac) 2 (1.9 mg, 0.0062 mmol, $10 \mathrm{~mol} \%$ ) was dissolved in $\mathrm{CH}_{2} \mathrm{Cl}_{2}(1 \mathrm{~mL})$ followed by stirring at room temperature under nitrogen atmosphere. After $1 \mathrm{~h}$, the solvent was distilled off and the resulting yellow Pd-complex was kept under vaccum for $10 \mathrm{~min}$. The remaining yellow solid was dissolved in $\mathrm{CHCl}_{3}(0.5 \mathrm{~mL})$ followed by adding of triflic acid (1.1 $\mu 1,0.012 \mathrm{mmol}, 20 \mathrm{~mol} \%)$ under nitrogen. The solution was stirred for few minutes until deep yellow color was observed. In other $10 \mathrm{ml}$ R.B. flask, a mixture of alkenyl oxime $1(0.124 \mathrm{mmol})$ and endione $2(0.062 \mathrm{mmol})$ was dissolved in $\mathrm{CHCl}_{3}(0.5 \mathrm{~mL})$ under nitrogen atmosphere. The resulting reaction mixture was transferred to the first flask that contains Pd-complex under nitrogen atmosphere. After the complete consumption of the endione 2 on TLC monitoring, the solvent was removed under vacuum. The remaining syrup was purified by silica gel column chromatography (Hex: $\left.\mathrm{Et}_{2} \mathrm{O}=1: 1\right)$ to give the pure domino products 3 .

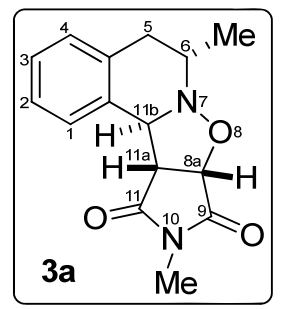

3a: White solid (quant., exo/endo $=94 / 6,8 \mathrm{~h})$; m.p. 175-176 ${ }^{\circ} \mathrm{C} ;{ }^{1} \mathrm{H}-\mathrm{NMR}\left(\mathrm{CDCl}_{3}\right)$ Major diastereomer: $\delta 7.47$ (d, 1H, ArH, $J=7.20 \mathrm{~Hz}$ ), 7.33-7.19 (m, 2H, ArH), 7.09 (d, 1H, ArH, $J=7.60 \mathrm{~Hz}), 4.85$ (d, 1H, H-8a, $J=7.20 \mathrm{~Hz}), 4.71$ (d, 1H, H-11b, $J=4.00 \mathrm{~Hz}), 3.72$ (dd, 1H, H-11a, $J=4.00,7.20 \mathrm{~Hz}$ ), 3.49-3.42 (m, 1H, H-6), 3.13-3.09 (m, 4H, H-5a, $\mathrm{NCH}_{3}$, $2.55(\mathrm{dd}, 1 \mathrm{H}, \mathrm{H}-5 \mathrm{~b}, J=6.00,16.40 \mathrm{~Hz}), 1.21\left(\mathrm{~d}, 3 \mathrm{H}, \mathrm{CH}_{3}, J=6.40\right)$; Minor diastereomer (representative peaks): $\delta 4.66(\mathrm{~d}, 1 \mathrm{H}, \mathrm{H}-11 \mathrm{~b}, J=7.20 \mathrm{~Hz}), 3.01\left(\mathrm{~s}, 3 \mathrm{H}, \mathrm{NCH}_{3}\right), 1.37(\mathrm{~d}$, $\left.3 \mathrm{H}, \mathrm{CH}_{3}, J=6.40 \mathrm{~Hz}\right) ;{ }^{13} \mathrm{C}-\mathrm{NMR}\left(\mathrm{CDCl}_{3}\right) \delta 175.7,174.0,132.8,132.7,128.9,127.6,127.5,127.1,76.0,64.2$, 56.7, 52.1, 32.0, 25.4, 18.0; enantiomeric excess: 70\%, determined by HPLC (Chiralpak IC, hexane/2-propanol $=5 / 1$, flow rate $=1 \mathrm{ml} / \mathrm{min}, 25^{\circ} \mathrm{C}, 214 \mathrm{~nm}$ ) minor peak: $\mathrm{t}_{\mathrm{R}}=26.6 \mathrm{~min}$, major peak: $\mathrm{t}_{\mathrm{R}}=16.5 \mathrm{~min} ;[\alpha]_{D}^{19}=$ $-91.8\left(\right.$ c $\left.0.85, \mathrm{CHCl}_{3}\right)$.

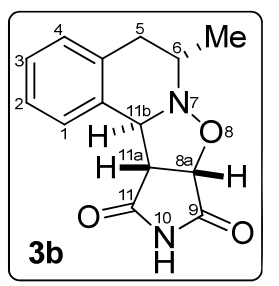

3b: White solid (quant., exo/endo $=93 / 7,12 \mathrm{~h})$; m.p. $150-151{ }^{\circ} \mathrm{C} ;{ }^{1} \mathrm{H}-\mathrm{NMR}\left(\mathrm{CDCl}_{3}\right)$ Major diastereomer: $\delta 8.12$ (brs, $1 \mathrm{H}, \mathrm{NH}$, hydrogen bonded), 7.43 (d, $1 \mathrm{H}, \mathrm{ArH}, J=7.20 \mathrm{~Hz}$ ), 7.29-7.21 (m, 2H, ArH), 7.10 (d, 1H, ArH, $J=7.20 \mathrm{~Hz}), 4.87$ (d, 1H, H-8a, $J=8.00 \mathrm{~Hz})$, $4.80(\mathrm{~d}, 1 \mathrm{H}, \mathrm{H}-11 \mathrm{~b}, J=3.60 \mathrm{~Hz}), 3.76(\mathrm{dd}, 1 \mathrm{H}, \mathrm{H}-11 \mathrm{a}, J=3.60,7.60 \mathrm{~Hz}), 3.50-3.44$ (m, 1H, H-6), 3.12 (dd, 1H, H-5a, J = 4.80), 2.64 (s, 1H, NH, non-hydrohen bonded), 2.57 (dd, $1 \mathrm{H}, \mathrm{H}-5 \mathrm{~b}, J=6.00,16.80 \mathrm{~Hz}), 1.24\left(\mathrm{~d}, 3 \mathrm{H}, \mathrm{CH}_{3} J=6.80\right.$ ); Minor diastereomer (representative peaks): $\delta 4.89$ (brs, 1H, H-8a), 4.70 (d, 1H, H-11b, J = 7.20 Hz), 3.80 (brs, 1H, H-11a), 3.31-3.27 (m, 1H, H-6), 3.02 (dd, 1H, $\mathrm{H}-5 \mathrm{a}, J=12.00,16.00), 2.51(\mathrm{dd}, 1 \mathrm{H}, \mathrm{H}-5 \mathrm{~b}, J=4.00,16.00 \mathrm{~Hz}), 1.40\left(\mathrm{~d}, 3 \mathrm{H}, \mathrm{CH}_{3}, J=6.40 \mathrm{~Hz}\right)$; ${ }^{13} \mathrm{C}-\mathrm{NMR}$ $\left(\mathrm{CDCl}_{3}\right) \delta 175.3,173.7,132.7,132.5,129.0,127.7,127.4,127.1,64.3,57.7,52.1,31.9,29.8,18.0$; HRMS (ESI) 
calcd for $\mathrm{C}_{14} \mathrm{H}_{14} \mathrm{~N}_{2} \mathrm{NaO}_{3}, \mathrm{~m} / \mathrm{z}=281.0897\left[(\mathrm{M}+\mathrm{Na})^{+}\right]$, found $\mathrm{m} / \mathrm{z}=281.0895$; enantiomeric excess: $68 \%$, determined by HPLC (Chiralpak IC, hexane $/ 2$-propanol $=5 / 1$, flow rate $=1 \mathrm{ml} / \mathrm{min}, 25^{\circ} \mathrm{C}, 214 \mathrm{~nm}$ ) minor peak: $\mathrm{t}_{\mathrm{R}}=28.6$ min, major peak: $\mathrm{t}_{\mathrm{R}}=19.3 \mathrm{~min} ;[\alpha]_{D}^{19}=-15.3\left(c\right.$ 0.49, $\left.\mathrm{CHCl}_{3}\right) ; \mathrm{IR}(\mathrm{KBr}): v 3171,3071,2920,1780$, $1707 \mathrm{~cm}^{-1}$.

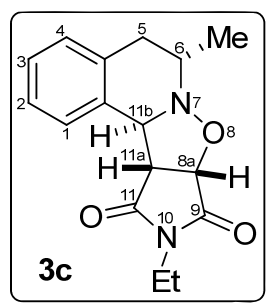

3c: White solid (quant., exo/endo $=97 / 3,8 \mathrm{~h}) ; 111-112{ }^{\circ} \mathrm{C} ;{ }^{1} \mathrm{H}-\mathrm{NMR}\left(\mathrm{CDCl}_{3}\right)$ Major diastereomer: $\delta 7.48(\mathrm{~d}, 1 \mathrm{H}, \mathrm{ArH}, J=7.60 \mathrm{~Hz}), 7.33-7.19(\mathrm{~m}, 2 \mathrm{H}, \mathrm{ArH}), 7.09(\mathrm{~d}, 1 \mathrm{H}, \mathrm{ArH}$, $J=7.20 \mathrm{~Hz}), 4.84$ (d, 1H, H-8a, $J=7.20 \mathrm{~Hz}), 4.71(\mathrm{~d}, 1 \mathrm{H}, \mathrm{H}-11 \mathrm{~b}, J=4.40 \mathrm{~Hz}), 3.71-3.62$ (m, 3H, H-11a, $\mathrm{CH}_{2} \mathrm{CH}_{3}$ ), 3.47-3.39 (m, 1H, H-6), 3.09 (dd, 1H, H-5a, J = 4.40, $16.40 \mathrm{~Hz}$ ), $2.57(\mathrm{dd}, 1 \mathrm{H}, \mathrm{H}-5 \mathrm{~b}, J=6.40,10.00 \mathrm{~Hz}), 1.25-1.21\left(\mathrm{~m}, 6 \mathrm{H}, \mathrm{CHCH}_{3}, \mathrm{CH}_{2} \underline{\mathrm{CH}_{3}}\right)$; Minor diastereomer (representative peaks): $\delta 4.63(\mathrm{~d}, 1 \mathrm{H}, \mathrm{H}-11 \mathrm{~b}, J=7.60 \mathrm{~Hz}), 3.28-3.22(\mathrm{~m}, 1 \mathrm{H}$, H-6), 3.01 (dd, 1H, H-5a, $J$ = 12.40, $16.40 \mathrm{~Hz}$ ), 2.49 (dd, 1H, H-5b, $J=4.00,16.40 \mathrm{~Hz}$ ), 1.37 (d, 3H, $\mathrm{CHCH}_{3}, J$ $=6.80 \mathrm{~Hz}) ;{ }^{13} \mathrm{C}-\mathrm{NMR}\left(\mathrm{CDCl}_{3}\right) \delta 175.5,173.7,132.8(2 \mathrm{C}), 128.8,127.6,127.5,127.1,76.0,64.4,56.6,52.1$, 34.3, 32.3, 18.1, 13.1; HRMS (ESI) calcd for $\mathrm{C}_{16} \mathrm{H}_{18} \mathrm{~N}_{2} \mathrm{NaO}_{3}, \mathrm{~m} / \mathrm{z}=309.1210\left[(\mathrm{M}+\mathrm{Na})^{+}\right]$, found $\mathrm{m} / \mathrm{z}=309.1207$; enantiomeric excess: $68 \%$, determined by HPLC (Chiralpak IC-3, hexane/2-propanol $=10 / 1$, flow rate $=1$ $\left.\mathrm{ml} / \mathrm{min}, 25^{\circ} \mathrm{C}, 214 \mathrm{~nm}\right)$ minor peak: $\mathrm{t}_{\mathrm{R}}=38.0$ min, major peak: $\mathrm{t}_{\mathrm{R}}=31.9 \mathrm{~min} ;[\alpha]_{D}^{19}=-34.4\left(c 0.41, \mathrm{CHCl}_{3}\right) ; \mathrm{IR}$ (KBr): $v 2987,2920,2851,1781,1694,1405 \mathrm{~cm}^{-1}$.

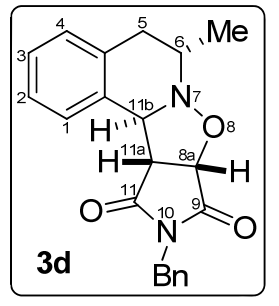

3d: White solid (quant., exo/endo $=95 / 5,8 \mathrm{~h}) ; 144-145{ }^{\circ} \mathrm{C} ;{ }^{1} \mathrm{H}-\mathrm{NMR}\left(\mathrm{CDCl}_{3}\right)$ Major diastereomer: $\delta 7.45(\mathrm{~d}, 1 \mathrm{H}, \mathrm{ArH}, J=7.20 \mathrm{~Hz}), 7.41-7.20(\mathrm{~m}, 7 \mathrm{H}, \mathrm{ArH}), 7.09(\mathrm{~d}, 1 \mathrm{H}, \mathrm{ArH}$, $J=7.20 \mathrm{~Hz}$ ), 4.87-4.64 (m, 4H, H-8a, H-11b, $\mathrm{NCH}_{2} \mathrm{Ph}$ ), 3.70 (dd, 1H, H-11a, $J=4.40$, $7.60 \mathrm{~Hz}$ ), 3.48-3.40 (m, 1H, H-6), 3.09 (dd, 1H, H-5a, $J=4.00,16.40 \mathrm{~Hz}$ ), 2.56 (dd, 1H, $\mathrm{H}-5 \mathrm{~b}, J=6.40,16.40 \mathrm{~Hz}), 1.22\left(\mathrm{~d}, 3 \mathrm{H}, \mathrm{CH}_{3}, J=6.40\right.$ ); Minor diastereomer (representative peaks): $\delta 1.38\left(\mathrm{~d}, 3 \mathrm{H}, \mathrm{CH}_{3}, J=6.40 \mathrm{~Hz}\right) ;{ }^{13} \mathrm{C}-\mathrm{NMR}\left(\mathrm{CDCl}_{3}\right) \delta 175.4,173.5,135.3,132.8,132.7,128.9(2 \mathrm{C})$, 128.8, 128.6 (2C), 128.2, 127.6, 127.5, 127.1, 76.1, 64.4, 56.6, 52.1, 42.8, 32.2, 18.1; HRMS (ESI) calcd for $\mathrm{C}_{21} \mathrm{H}_{20} \mathrm{~N}_{2} \mathrm{NaO}_{3}, \mathrm{~m} / \mathrm{z}=371.1366\left[(\mathrm{M}+\mathrm{Na})^{+}\right]$, found $\mathrm{m} / \mathrm{z}=371.1362$; enantiomeric excess: $70 \%$, determined by HPLC (Chiralcel IC-3, hexane $/ 2$-propanol $=10 / 1$, flow rate $=1 \mathrm{ml} / \mathrm{min}, 25^{\circ} \mathrm{C}, 214 \mathrm{~nm}$ ) minor peak: $t_{\mathrm{R}}=38.4$ min, major peak: $t_{\mathrm{R}}=32.4 \mathrm{~min} ;[\alpha]_{D}^{18}=-26.8\left(c 0.69, \mathrm{CHCl}_{3}\right)$; IR (KBr): $v$ 2961, 2926, 2853, 1776, 1704, $1496,1456,1433 \mathrm{~cm}^{-1}$. 


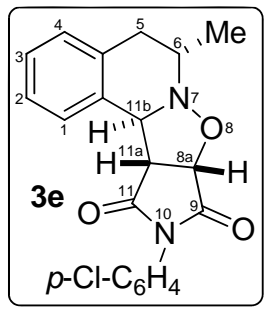

3e: Yellow solid (quant., exo/endo $=93 / 7,4 \mathrm{~h}) ; 175-176{ }^{\circ} \mathrm{C}$; ${ }^{1} \mathrm{H}-\mathrm{NMR}\left(\mathrm{CDCl}_{3}\right)$ Major diastereomer: $\delta$ 7.49-7.46 (m, 3H, ArH), 7.37-7.22 (m, 4H, ArH), 7.12 (d, 1H, ArH, $J=$ $7.60 \mathrm{~Hz}), 4.99$ (d, 1H, H-8a, $J=8.00 \mathrm{~Hz}), 4.86(\mathrm{~d}, 1 \mathrm{H}, \mathrm{H}-11 \mathrm{~b}, J=3.60 \mathrm{~Hz}), 3.88$ (dd, 1H, H-11a, $J=4.40,8 \mathrm{~Hz}), 3.55-3.47$ (m, 1H, H-6), 3.15 (dd, 1H, H-5a, $J=4.80,16.80 \mathrm{~Hz}$ ), 2.59 (dd, $1 \mathrm{H}, \mathrm{H}-5 \mathrm{~b}, J=6.00,16.80 \mathrm{~Hz}), 1.26\left(\mathrm{~d}, 3 \mathrm{H}, \mathrm{CH}_{3} J=6.40\right)$; Minor diastereomer (representative peaks): 4.96 (brs, 1H, H-8a), 4.81 (d, 1H, H-11b, $J=8.00 \mathrm{~Hz}$ ), 3.33-3.28 $\left.\mathrm{CH}_{3}, J=7.20 \mathrm{~Hz}\right) ;{ }^{13} \mathrm{C}-\mathrm{NMR}\left(\mathrm{CDCl}_{3}\right) \delta 163.6,161.7,123.9,121.8,121.5,118.9,118.63(2 \mathrm{C}), 118.56,118.0$, 116.8, 116.4, 116.2, 64.8, 53.7, 45.6, 41.2, 21.0, 7.1; HRMS (ESI) calcd for $\mathrm{C}_{20} \mathrm{H}_{17} \mathrm{ClN}_{2} \mathrm{NaO}_{3}, \mathrm{~m} / \mathrm{z}=391.0820$ $\left[(\mathrm{M}+\mathrm{Na})^{+}\right]$, found $\mathrm{m} / \mathrm{z}=391.0817$; enantiomeric excess: $70 \%$, determined by HPLC (Chiralpak IE, hexane $/ 2$-propanol $=10 / 1$, flow rate $1=\mathrm{ml} / \mathrm{min}, 25^{\circ} \mathrm{C}, 214 \mathrm{~nm}$ ) minor peak: $t_{R}=53.3 \mathrm{~min}$, major peak: $t_{R}=39.4$ $\min ;[\alpha]_{D}^{24}=-29.5\left(c 0.57, \mathrm{CHCl}_{3}\right)$; IR (KBr): v 3011, 2930, 2855, 1782, 1713, 1496, $1456 \mathrm{~cm}^{-1}$.

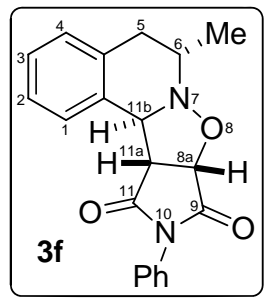

3f: Pale yellow solid (quant., exo/endo $=94 / 6,4 \mathrm{~h}) ; 157-158{ }^{\circ} \mathrm{C} ;{ }^{1} \mathrm{H}-\mathrm{NMR}\left(\mathrm{CDCl}_{3}\right)$ Major diasteromer: $\delta$ 7.53-7.49 (m, 3H, ArH), 7.46-7.42 (m, 1H, ArH), 7.39-7.36 (m, 2H, ArH), 7.31-7.23 (m, 2H, ArH), 7.12 (d, 1H, ArH, $J=8.00 \mathrm{~Hz}$ ), 5.01 (d, 1H, H-8a, $J=7.60 \mathrm{~Hz}$ ), $4.88(\mathrm{~d}, 1 \mathrm{H}, \mathrm{H}-11 \mathrm{~b}, J=4.00 \mathrm{~Hz}), 3.88(\mathrm{dd}, 1 \mathrm{H}, \mathrm{H}-11 \mathrm{a}, J=4.40,7.60 \mathrm{~Hz}), 3.53-3.46$ (m, $1 \mathrm{H}, \mathrm{H}-6), 3.13$ (dd, 1H, H-5a, $J=4.40,16.40 \mathrm{~Hz}$ ), 2.61 (dd, $1 \mathrm{H}, \mathrm{H}-5 \mathrm{~b}, J=6.40,18.80 \mathrm{~Hz}$ ), 1.27 (d, $3 \mathrm{H}, \mathrm{CH}_{3,} J=6.40$ ); Minor diasteromer (representative peaks): $\delta 4.97$ (brs, $1 \mathrm{H}, \mathrm{H}-8 \mathrm{a}$ ), 4.81 (d, $1 \mathrm{H}, \mathrm{H}-11 \mathrm{~b}$, $J=7.60 \mathrm{~Hz}$ ), 3.33-3.28 (m, 1H, H-6), 3.05 (dd, 1H, H-5a, $J=12.00,16.80 \mathrm{~Hz}$ ), 2.53 (dd, 1H, H-5b, $J=4.00$, $16.40 \mathrm{~Hz}), 1.41\left(\mathrm{~d}, 3 \mathrm{H}, \mathrm{CH}_{3}, J=6.40 \mathrm{~Hz}\right) ;{ }^{13} \mathrm{C}-\mathrm{NMR}\left(\mathrm{CDCl}_{3}\right) \delta 174.8,172.8,132.9,132.7,129.4(2 \mathrm{C}), 129.1$, 128.9, 127.7, 127.6, 127.2, 126.5 (2C), 76.0, 64.9, 56.7, 52.3, 32.3, 29.9, 18.2; HRMS (ESI) calcd for $\mathrm{C}_{20} \mathrm{H}_{18} \mathrm{~N}_{2} \mathrm{NaO}_{3}, \mathrm{~m} / \mathrm{z}=357.1210\left[(\mathrm{M}+\mathrm{Na})^{+}\right]$, found $\mathrm{m} / \mathrm{z}=357.1206$; enantiomeric excess: $70 \%$, determined by HPLC (Chiralpak IE, hexane $/ 2$-propanol $=10 / 1$, flow rate $=1 \mathrm{ml} / \mathrm{min}, 25^{\circ} \mathrm{C}, 214 \mathrm{~nm}$ ) minor peak: $t_{R}=69.9 \mathrm{~min}$, major peak: $\mathrm{t}_{\mathrm{R}}=56.5 \mathrm{~min} ;[\alpha]_{D}^{23}=-34.4\left(\mathrm{c} 0.52, \mathrm{CHCl}_{3}\right) ; \mathrm{IR}(\mathrm{KBr}): v 2927,2854,1778,1717,1498,1456$ $\mathrm{cm}^{-1}$.

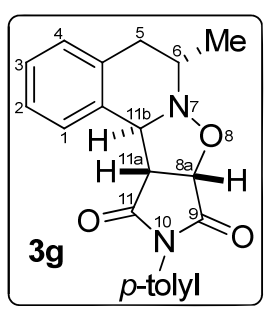

3g: Pale yellow solid (quant., exo/endo $=94 / 6,4 \mathrm{~h}) ; 162-163{ }^{\circ} \mathrm{C} ;{ }^{1} \mathrm{H}-\mathrm{NMR}\left(\mathrm{CDCl}_{3}\right)$ Major diastereomer: $\delta 7.51(\mathrm{~d}, 1 \mathrm{H}, \mathrm{ArH}, J=7.20 \mathrm{~Hz}), 7.32-7.21(\mathrm{~m}, 6 \mathrm{H}, \mathrm{ArH}), 7.11(\mathrm{~d}, 1 \mathrm{H}, \mathrm{ArH}$, $J=8.00 \mathrm{~Hz}), 4.99(\mathrm{~d}, 1 \mathrm{H}, \mathrm{H}-8 \mathrm{a}, J=7.60 \mathrm{~Hz}), 4.86(\mathrm{~d}, 1 \mathrm{H}, \mathrm{H}-11 \mathrm{~b}, J=4.00 \mathrm{~Hz}), 3.86(\mathrm{dd}$, $1 \mathrm{H}, \mathrm{H}-11 \mathrm{a}, J=4.40,7.60 \mathrm{~Hz}$ ), 3.51-3.44 (m, 1H, H-6), 3.12 (dd, 1H, H-5a, $J=4.80,16.40$ $\mathrm{Hz}), 2.61(\mathrm{dd}, 1 \mathrm{H}, \mathrm{H}-5 \mathrm{~b}, J=6.40,16.80 \mathrm{~Hz}), 2.40\left(\mathrm{~s}, 3 \mathrm{H}, \mathrm{C}_{6} \mathrm{H}_{4}-\mathrm{CH}_{3}\right), 1.27$ (d, $3 \mathrm{H}, \mathrm{CH}_{3} J=$ 6.40); Minor diastereomer (representative peaks): $\delta 7.37$ (d, $1 \mathrm{H}, \mathrm{ArH}, J=7.60 \mathrm{~Hz}$ ), 4.96 (brs, 1H, H-8a), 4.79 (d, 1H, H-11b, $J=8.00$ Hz), 3.32-3.28 (m, 1H, H-6), 3.05 (dd, 1H, H-5a, $J=11.60,16.40$ $\mathrm{Hz}$ ), $2.52(\mathrm{dd}, 1 \mathrm{H}, \mathrm{H}-5 \mathrm{~b}, J=4.00,16.40 \mathrm{~Hz}), 1.41\left(\mathrm{~d}, 3 \mathrm{H}, \mathrm{CH}_{3}, J=6.80 \mathrm{~Hz}\right) ;{ }^{13} \mathrm{C}-\mathrm{NMR}\left(\mathrm{CDCl}_{3}\right) \delta 174.9,172.9$, 
$139.2,132.9,132.8,130.0,128.9,128.8,127.7,127.6,127.1,126.3,76.0,65.0,56.6,52.2,32.4,21.4,18.2$; HRMS (ESI) calcd for $\mathrm{C}_{21} \mathrm{H}_{20} \mathrm{~N}_{2} \mathrm{NaO}_{3}, \mathrm{~m} / \mathrm{z}=371.1366\left[(\mathrm{M}+\mathrm{Na})^{+}\right]$, found $\mathrm{m} / \mathrm{z}=371.1363$; enantiomeric excess: $70 \%$, determined by HPLC (Chiralpak IC-3, hexane/2-propanol $=10 / 1$, flow rate $=1 \mathrm{ml} / \mathrm{min}, 25^{\circ} \mathrm{C}, 214 \mathrm{~nm}$ ) minor peak: $\mathrm{t}_{\mathrm{R}}=49.1 \mathrm{~min}$, major peak: $\mathrm{t}_{\mathrm{R}}=53.8 \mathrm{~min} ;[\alpha]_{D}^{25}=-43.1\left(\mathrm{c} 1.08, \mathrm{CHCl}_{3}\right)$; IR $(\mathrm{KBr}): v 2924,2882$, $2853,1781,1719,1516,1793,1455 \mathrm{~cm}^{-1}$.

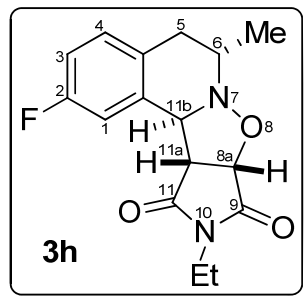

3h: White solid $(92 \%$, exo/endo $=96 / 4,12 \mathrm{~h}) ; 138-139{ }^{\circ} \mathrm{C} ;{ }^{1} \mathrm{H}-\mathrm{NMR}\left(\mathrm{CDCl}_{3}\right)$ Major diastereomer: $\delta 7.20(\mathrm{dd}, 1 \mathrm{H}, \mathrm{H}-1, J=2.40,9.20 \mathrm{~Hz}), 7.06(\mathrm{dd}, 1 \mathrm{H}, \mathrm{H}-4, J=5.60,8.40$ $\mathrm{Hz}), 6.93(\mathrm{td}, 1 \mathrm{H}, \mathrm{H}-3, J=2.00,8.00 \mathrm{~Hz}), 4.83$ (d, 1H, H-8a, J = 7.20 Hz), 4.66 (d, 1H, $\mathrm{H}-11 \mathrm{~b}, J=3.60 \mathrm{~Hz}$ ), 3.68-3.62 (m, 3H, H-11a, $\left.\underline{\mathrm{CH}_{2}} \mathrm{CH}_{3}\right), 3.49-3.41$ (m, 1H, H-6), 3.05 $(\mathrm{dd}, 1 \mathrm{H}, \mathrm{H}-5 \mathrm{a}, J=4.00,16.40 \mathrm{~Hz}), 2.52(\mathrm{dd}, 1 \mathrm{H}, \mathrm{H}-5 \mathrm{~b}, J=6.40,16.40 \mathrm{~Hz}), 1.25-1.20$ (m, $6 \mathrm{H}, \mathrm{CHCH}_{3}, \mathrm{CH}_{2} \underline{\mathrm{CH}}_{3}$ ); Minor diastereomer $\mathrm{z}$ (representative peaks): $\delta 4.79$ (brs, $1 \mathrm{H}$, H-8a), 4.63 (brs, 1H, H-11b), 1.37 (d, 3H, $\left.\mathrm{CHCH}_{3}, J=6.40 \mathrm{~Hz}\right) ;{ }^{13} \mathrm{C}-\mathrm{NMR}\left(\mathrm{CDCl}_{3}\right) \delta 175.3,173.6,161.7(\mathrm{~d}$, $\left.{ }^{1} J_{\mathrm{C}-\mathrm{F}}=245.0 \mathrm{~Hz}\right), 134.6\left(\mathrm{~d},{ }^{3} J_{\mathrm{C}-\mathrm{F}}=6.7 \mathrm{~Hz}\right), 130.5\left(\mathrm{~d},{ }^{3} J_{\mathrm{C}-\mathrm{F}}=7.6 \mathrm{~Hz}\right), 128.4\left(\mathrm{~d},{ }^{4} J_{\mathrm{C}-\mathrm{F}}=2.9 \mathrm{~Hz}\right), 115.0\left(\mathrm{~d},{ }^{2} J_{\mathrm{C}-\mathrm{F}}=\right.$ $21.0 \mathrm{~Hz}), 113.9\left(\mathrm{~d},{ }^{2} J_{\mathrm{C}-\mathrm{F}}=21.9 \mathrm{~Hz}\right), 75.8,64.1,56.4,52.1,34.4,31.3,18.0,13.1 ;{ }^{19} \mathrm{~F}-\mathrm{NMR}\left(\mathrm{CDCl}_{3}\right): \delta-114.7$; HRMS (ESI) calcd for $\mathrm{C}_{16} \mathrm{H}_{17} \mathrm{FN}_{2} \mathrm{NaO}_{3}, \mathrm{~m} / \mathrm{z}=327.1115\left[(\mathrm{M}+\mathrm{Na})^{+}\right]$, found $\mathrm{m} / \mathrm{z}=327.1111$; enantiomeric excess: $60 \%$, determined by HPLC (Chiralpak IF, hexane/2-propanol $=5 / 1$, flow rate $1 \mathrm{ml} / \mathrm{min}, 25^{\circ} \mathrm{C}, 214 \mathrm{~nm}$ ) minor peak: $t_{\mathrm{R}}=44.9 \min$, major peak: $\mathrm{t}_{\mathrm{R}}=31.2 \mathrm{~min} ;[\alpha]_{D}^{25}=-30.4\left(c\right.$ 0.25, $\left.\mathrm{CHCl}_{3}\right)$; IR $(\mathrm{KBr}): v 2997,2925,2897$, $2851,1780,1712,1616,1503,1404 \mathrm{~cm}^{-1}$.

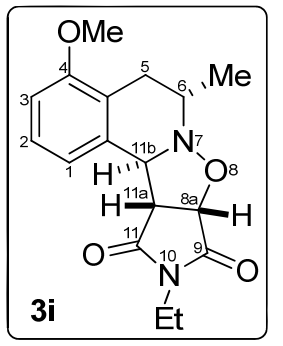

3i: White solid $(92 \%$, exo/endo $=95 / 5,12 \mathrm{~h}) ; 147-148{ }^{\circ} \mathrm{C} ;{ }^{1} \mathrm{H}-\mathrm{NMR}\left(\mathrm{CDCl}_{3}\right)$ Major diastereomer: $\delta$ 7.27-7.23 (m, 1H, H-2), 7.1 (d, 1H, H-1, J = 7.60 Hz), 6.76 (d, 1H, H-3, J $=8.40 \mathrm{~Hz}), 4.84(\mathrm{~d}, 1 \mathrm{H}, \mathrm{H}-8 \mathrm{a}, J=8.00 \mathrm{~Hz}), 4.68(\mathrm{~d}, 1 \mathrm{H}, \mathrm{H}-11 \mathrm{~b}, J=4.00 \mathrm{~Hz}), 3.83$ (s, 3H, $\mathrm{OCH}_{3}$ ), 3.70-3.62 (m, 3H, H-11a, $\mathrm{CH}_{2} \mathrm{CH}_{3}$ ), 3.42-3.34 (m, 1H, H-6), 2.99 (dd, 1H, H-5a, J $=4.40,17.20 \mathrm{~Hz}), 2.48(\mathrm{dd}, 1 \mathrm{H}, \mathrm{H}-5 \mathrm{~b}, J=6.40,17.20 \mathrm{~Hz}), 1.24-1.21\left(\mathrm{~m}, 6 \mathrm{H}, \mathrm{CHCH}_{3}\right.$, $\mathrm{CH}_{2} \mathrm{CH}_{3}$ ); Minor diastereomer (representative peaks): $\delta 1.39$ (d, $3 \mathrm{H}, \mathrm{CHCH}_{3}, J=6.80 \mathrm{~Hz}$ ); ${ }^{13} \mathrm{C}-\mathrm{NMR}\left(\mathrm{CDCl}_{3}\right) \delta 175.5,173.6,156.7,133.8,127.6,121.9,119.4,108.6,76.0,64.4,56.5,55.5,51.7,34.3$, 26.6, 18.4, 13.1; HRMS (ESI) calcd for $\mathrm{C}_{17} \mathrm{H}_{20} \mathrm{~N}_{2} \mathrm{NaO}_{4}, \mathrm{~m} / \mathrm{z}=339.1315\left[(\mathrm{M}+\mathrm{Na})^{+}\right]$, found $\mathrm{m} / \mathrm{z}=339.1313$; enantiomeric excess: $52 \%$, determined by HPLC (Chiralpak IF, hexane/2-propanol = 5/1, flow rate $1 \mathrm{ml} / \mathrm{min}$, $\left.25^{\circ} \mathrm{C}, 214 \mathrm{~nm}\right)$ minor peak: $\mathrm{t}_{\mathrm{R}}=134.3 \mathrm{~min}$, major peak: $\mathrm{t}_{\mathrm{R}}=26.4 \mathrm{~min} ;[\alpha]_{D}^{19}=-27.1\left(\mathrm{c} 0.41, \mathrm{CHCl}_{3}\right) ; \mathrm{IR}$ $(\mathrm{KBr}): v 3015,2976,2856,1772,1713,1593,1472,1401 \mathrm{~cm}^{-1}$. 


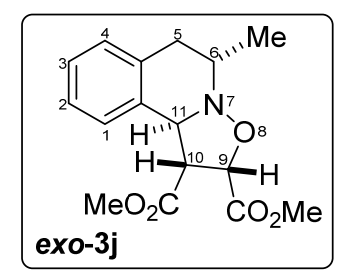

3j: (quant., exo/endo $=1 / 1,12 \mathrm{~h})$; exo-3j: White solid; $150-151{ }^{\circ} \mathrm{C} ;{ }^{1} \mathrm{H}-\mathrm{NMR}\left(\mathrm{CDCl}_{3}\right) \delta$ $7.21(\mathrm{t}, 1 \mathrm{H}, \mathrm{ArH}, J=7.20 \mathrm{~Hz}), 7.17$ (t, 1H, ArH, $J=7.20 \mathrm{~Hz}), 7.10$ (d, 1H, ArH, $J=$ $7.20 \mathrm{~Hz}$ ), 7.07 (d, 1H, ArH, J = 7.20 Hz), 5.00 (2 overlapping d, 2H, H-9, H-11), 3.80 $\left(\mathrm{s}, 3 \mathrm{H}, \mathrm{COOCH}_{3}\right), 3.79$ (s, $\left.3 \mathrm{H}, \mathrm{COOCH}_{3}\right), 3.72(\mathrm{t}, 1 \mathrm{H}, \mathrm{H}-10, J=9.60 \mathrm{~Hz}), 3.09-3.06$ (m, 1H, H-6), 2.86 (dd, 1H, H-5a, $J=4.20,16.20 \mathrm{~Hz}$ ), 2.80 (dd, 1H, H-5b, $J=10.20$, $16.80 \mathrm{~Hz}), 1.34\left(\mathrm{~d}, 3 \mathrm{H}, \mathrm{CHCH}_{3}, J=6.00 \mathrm{~Hz}\right) ;{ }^{13} \mathrm{C}-\mathrm{NMR}\left(\mathrm{CDCl}_{3}\right) \delta 170.8,169.6,133.2,133.0,128.3,127.7$, 126.9, 126.7, 77.9, 66.7, 57.9, 53.1, 52.8, 52.7, 36.9, 19.1; HRMS (ESI) calcd for $\mathrm{C}_{16} \mathrm{H}_{19} \mathrm{NNaO}_{5}, \mathrm{~m} / \mathrm{z}=328.1155$ $\left[(\mathrm{M}+\mathrm{Na})^{+}\right]$, found $\mathrm{m} / \mathrm{z}=328.1151$; enantiomeric excess: $68 \%$, determined by HPLC (Chiralpak IF, hexane $/ 2$-propanol $=5 / 1$, flow rate $1.0 \mathrm{ml} / \mathrm{min}, 25^{\circ} \mathrm{C}, 214 \mathrm{~nm}$ ) minor peak: $t_{R}=73.8$ min, major peak: $t_{R}=26.2$ $\min ;[\alpha]_{D}^{18}=-84\left(\right.$ c $\left.0.05, \mathrm{CHCl}_{3}\right) ; \mathrm{IR}(\mathrm{KBr}): v 2923,2852,1752,1725,1439 \mathrm{~cm}^{-1}$.

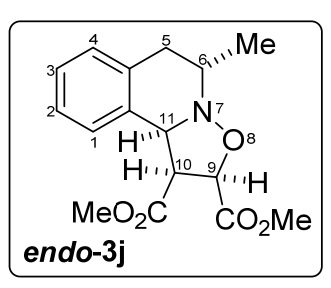

endo-3j: White solid; 116-117 ${ }^{\circ} \mathrm{C} ;{ }^{1} \mathrm{H}-\mathrm{NMR}\left(\mathrm{CDCl}_{3}\right) \delta$ 7.17-7.13 (m, 2H, $\left.\mathrm{ArH}\right)$, 7.10-7.06 (m, 2H, ArH), 5.03 (d, 1H, H-9, $J=9.60 \mathrm{~Hz}$ ), 5.01 (d, 1H, H-11, $J=10.80$ $\mathrm{Hz}$ ), 4.27 (t, 1H, H-10, $J=9.60 \mathrm{~Hz}$ ), 3.87-3.84 (m, 1H, H-6), 3.70 (s, 3H, $\mathrm{COOCH}_{3}$ ), $3.21\left(\mathrm{~s}, 3 \mathrm{H}, \mathrm{COOCH}_{3}\right), 2.82$ (dd, $1 \mathrm{H}, \mathrm{H}-5 \mathrm{a}, J=3.60,16.20 \mathrm{~Hz}$ ), 2.70 (dd, $1 \mathrm{H}, \mathrm{H}-5 \mathrm{~b}, J$

$=10.80,16.20 \mathrm{~Hz}), 1.46\left(\mathrm{~d}, 3 \mathrm{H}, \mathrm{CHCH} \underline{3}_{3}, J=6.60 \mathrm{~Hz}\right) ;{ }^{13} \mathrm{C}-\mathrm{NMR}\left(\mathrm{CDCl}_{3}\right) \delta 171.1$, 170.8, 134.7, 130.7, 128.0, 127.7, 127.5, 125.6, 80.0, 66.4, 57.8, 52.4, 51.9, 51.8, 37.1, 19.9; HRMS (ESI) calcd for $\mathrm{C}_{16} \mathrm{H}_{19} \mathrm{NNaO}_{5}, \mathrm{~m} / \mathrm{z}=328.1155\left[(\mathrm{M}+\mathrm{Na})^{+}\right]$, found $\mathrm{m} / \mathrm{z}=328.1151$; enantiomeric excess: $68 \%$, determined by HPLC (Chiralpak IF, hexane/2-propanol $=5 / 1$, flow rate $1.0 \mathrm{ml} / \mathrm{min}, 25^{\circ} \mathrm{C}, 214 \mathrm{~nm}$ ) minor peak: $t_{R}=12.4 \mathrm{~min}$, major peak: $\mathrm{t}_{\mathrm{R}}=21.2 \mathrm{~min} ;[\alpha]_{D}^{18}=+40\left(\right.$ c 0.1, $\left.\mathrm{CHCl}_{3}\right)$; IR $(\mathrm{KBr}): v 2925,2851,1762,1730,1436 \mathrm{~cm}^{-1}$.

\section{E. References}

1. Y. Hoshimoto, Y. Hayashi, H. Suzuki, M. Ohashi and S. Ogoshi, Angew. Chem. 2012, 124, 10970.

2. S. J. Danishcfsky, P. J. Harrison, R. R. Webb and B. T. O'Neill, J. Am. Chem. Soc. 1985, 107, 1421.

3. M. Frederickson, R. Grigg, J. Markandu, M. Thornton-Pett and J. Redpath, Tetrahedron 1997, 53, 15051. 


\section{F. NMR charts}
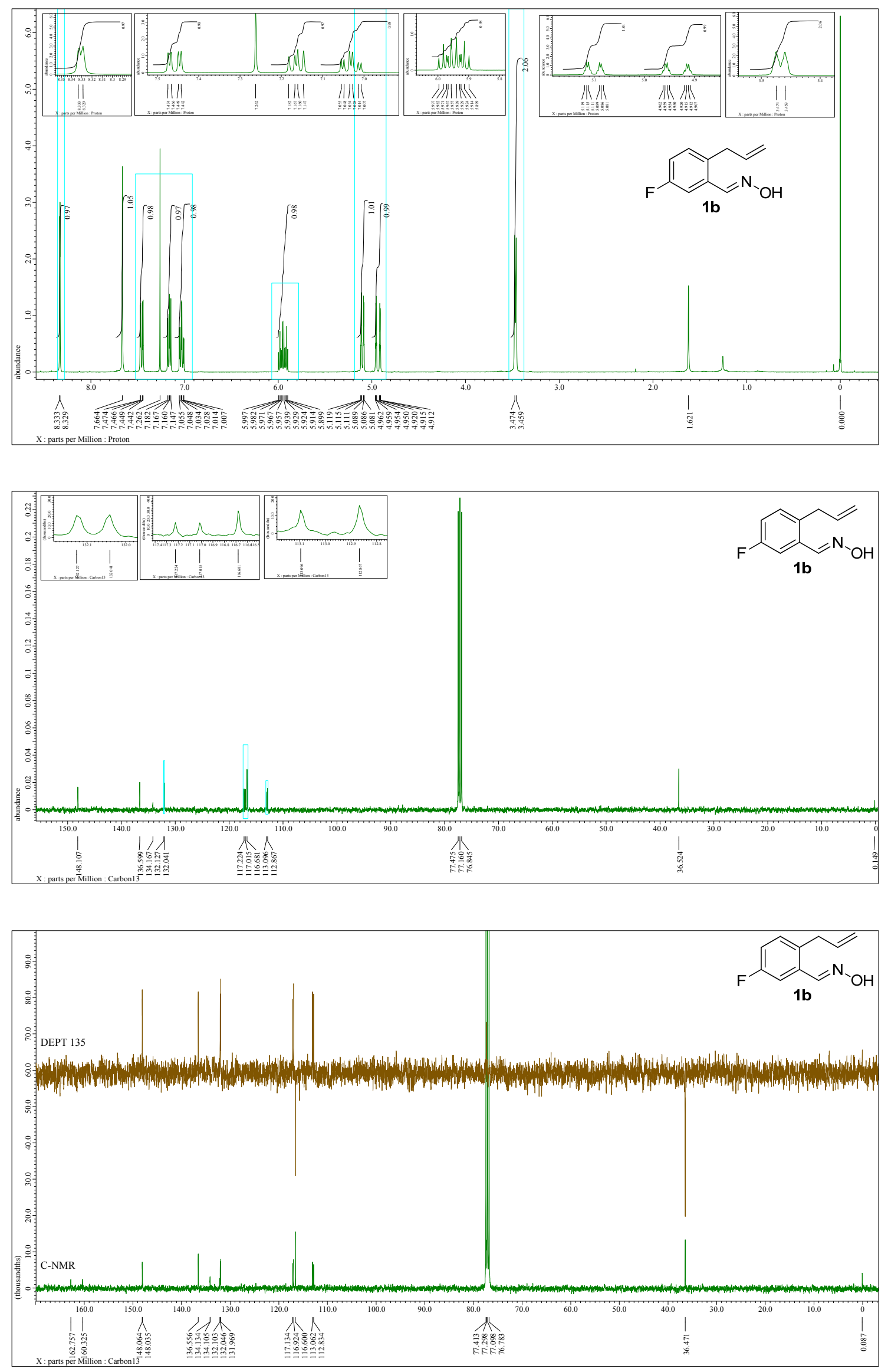

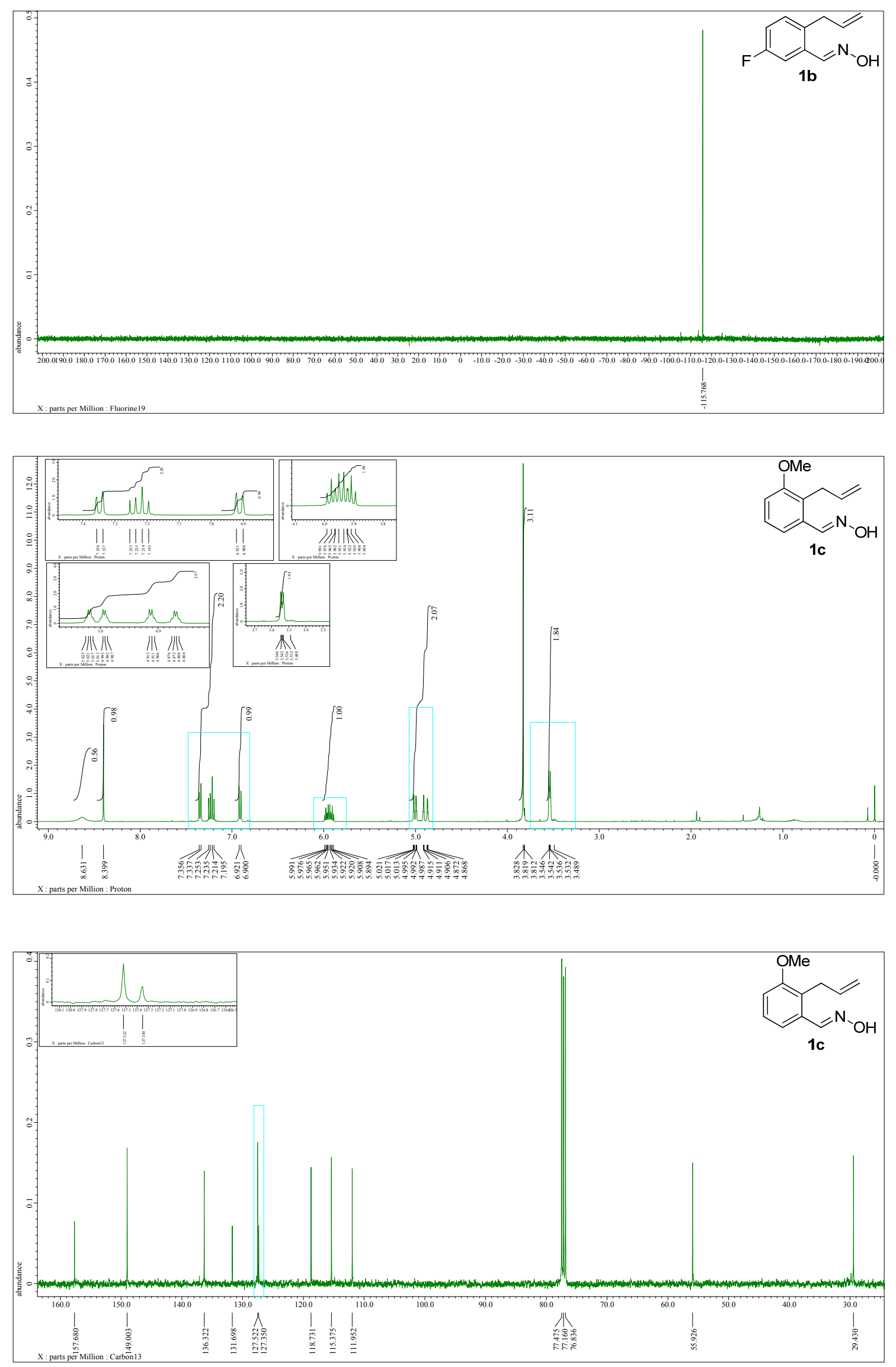

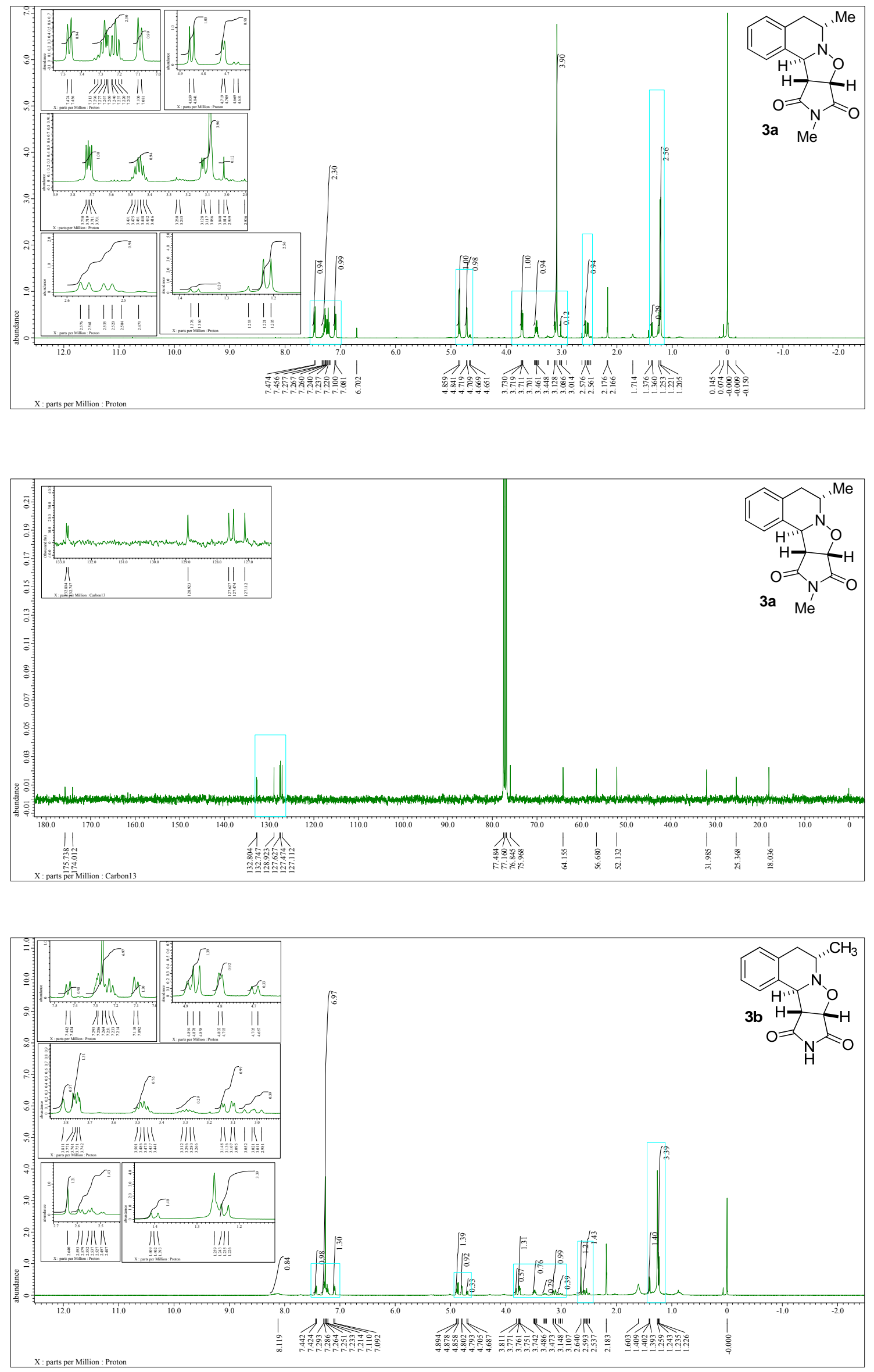

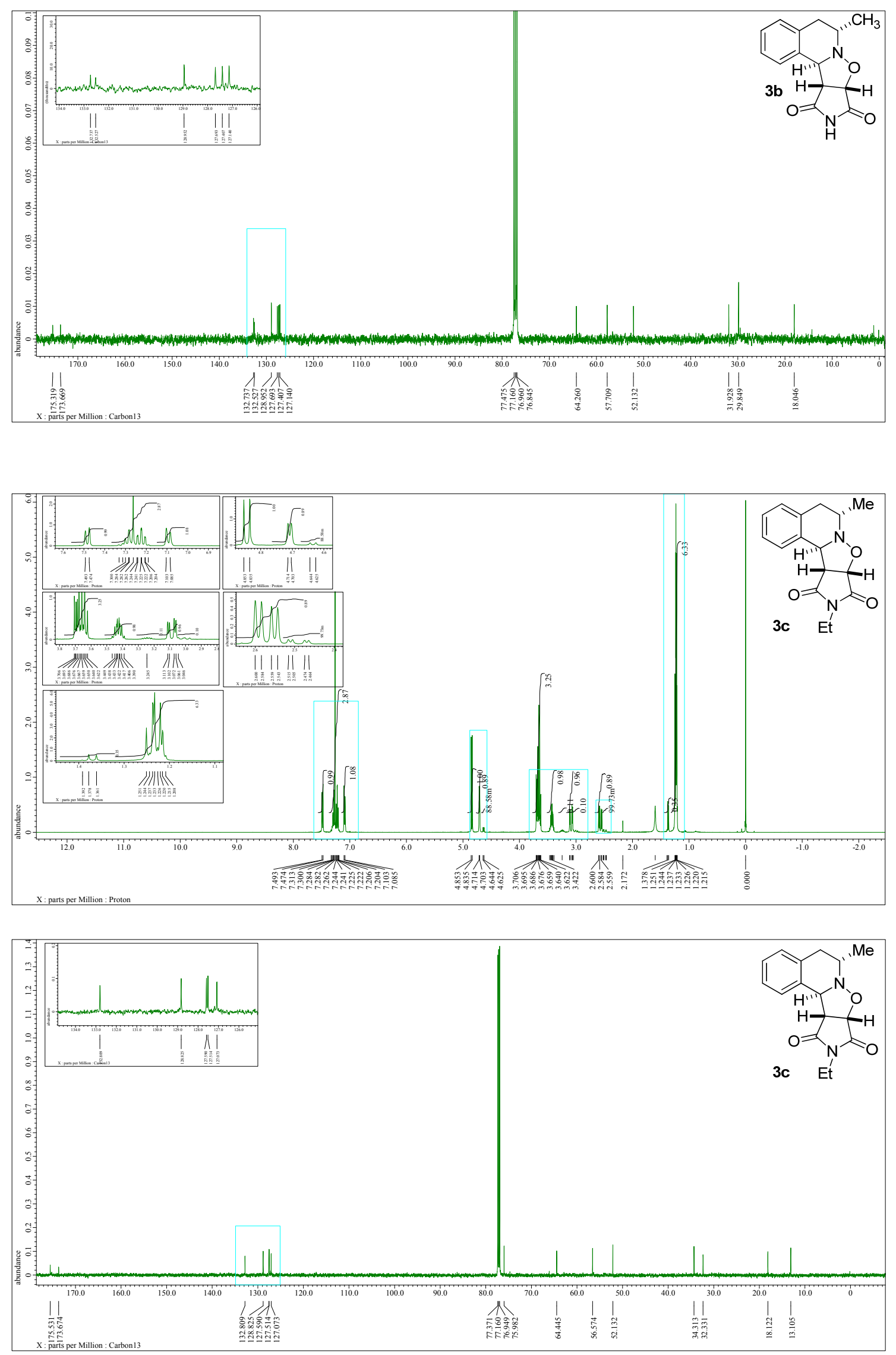

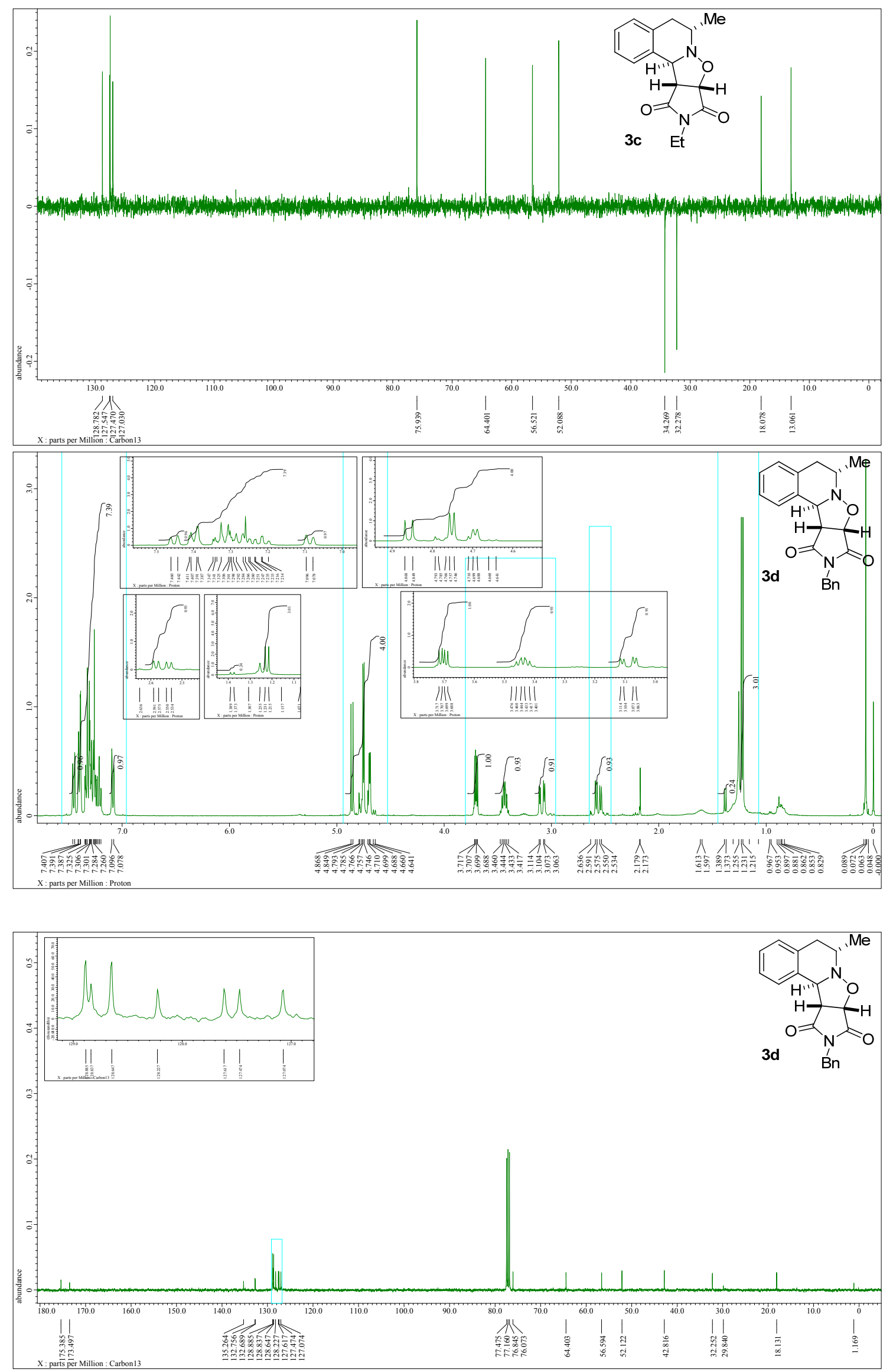

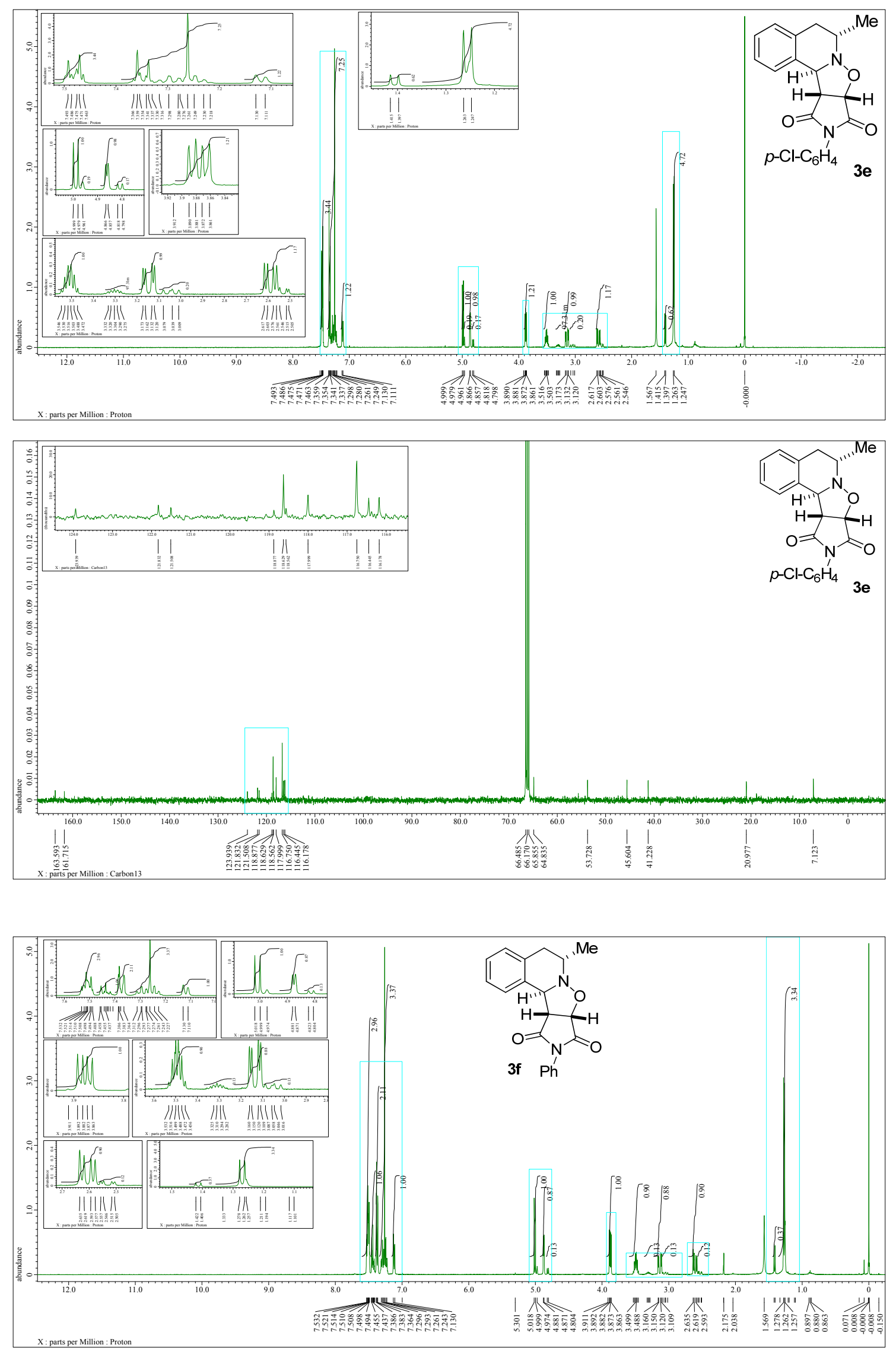

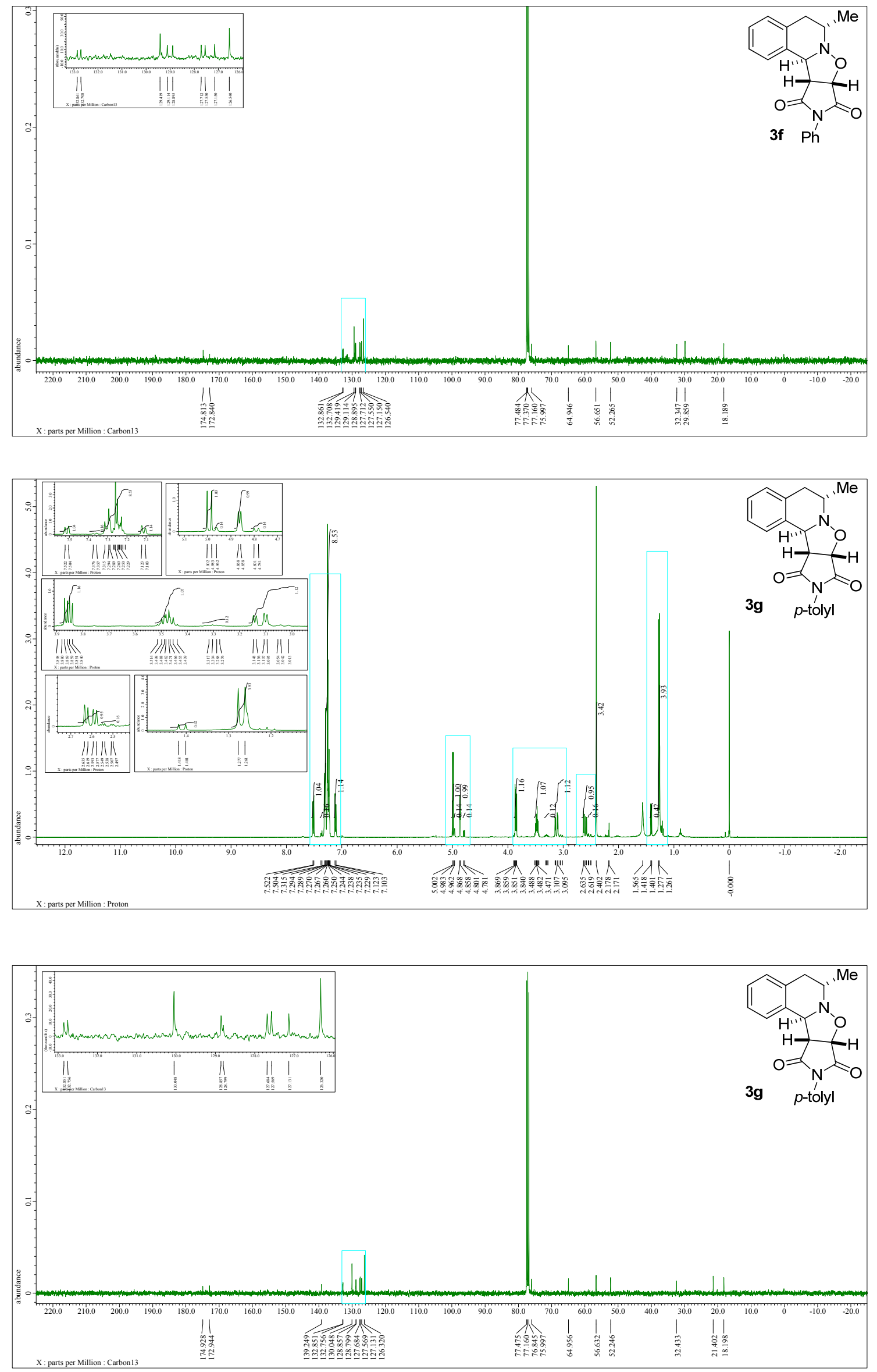

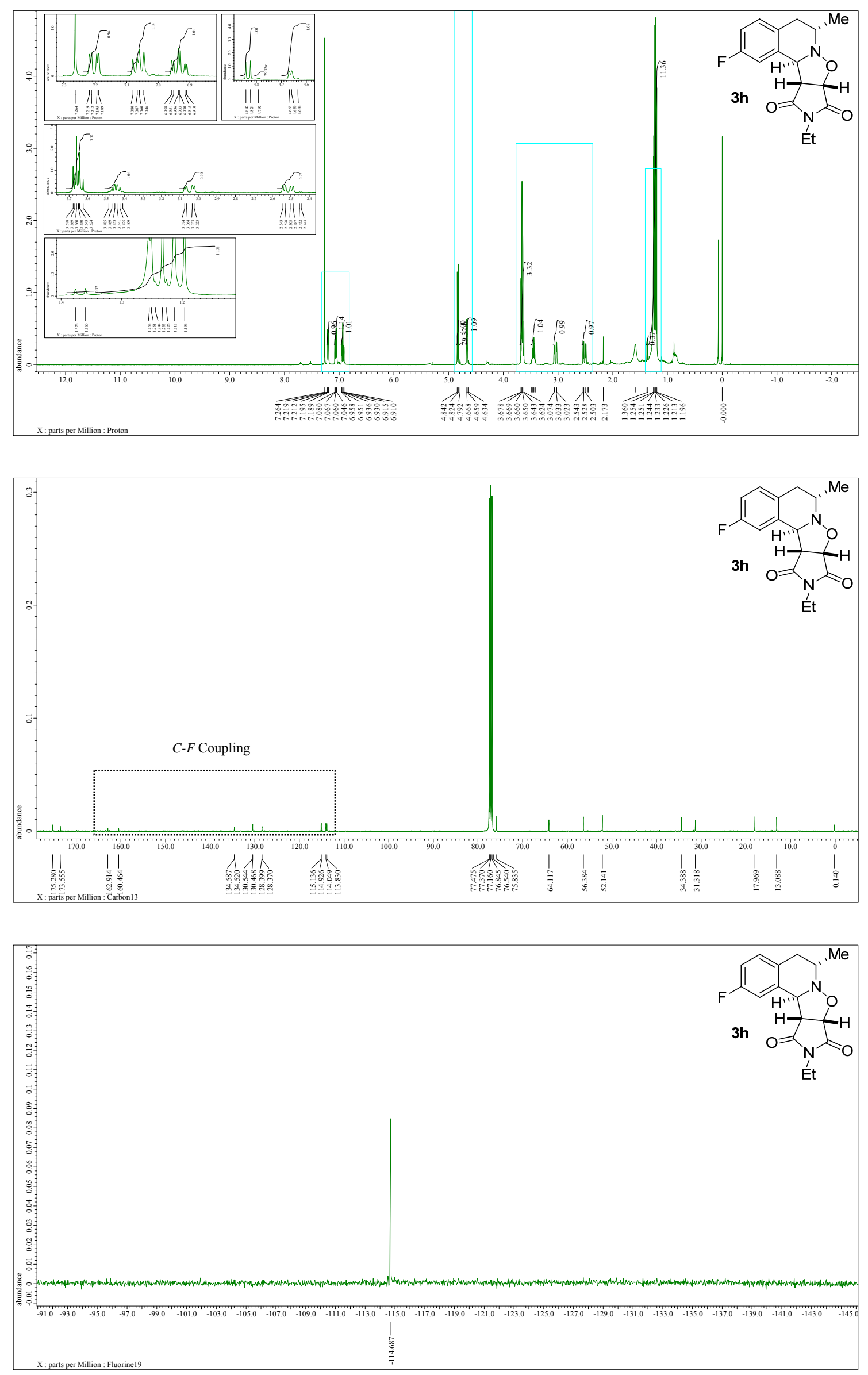

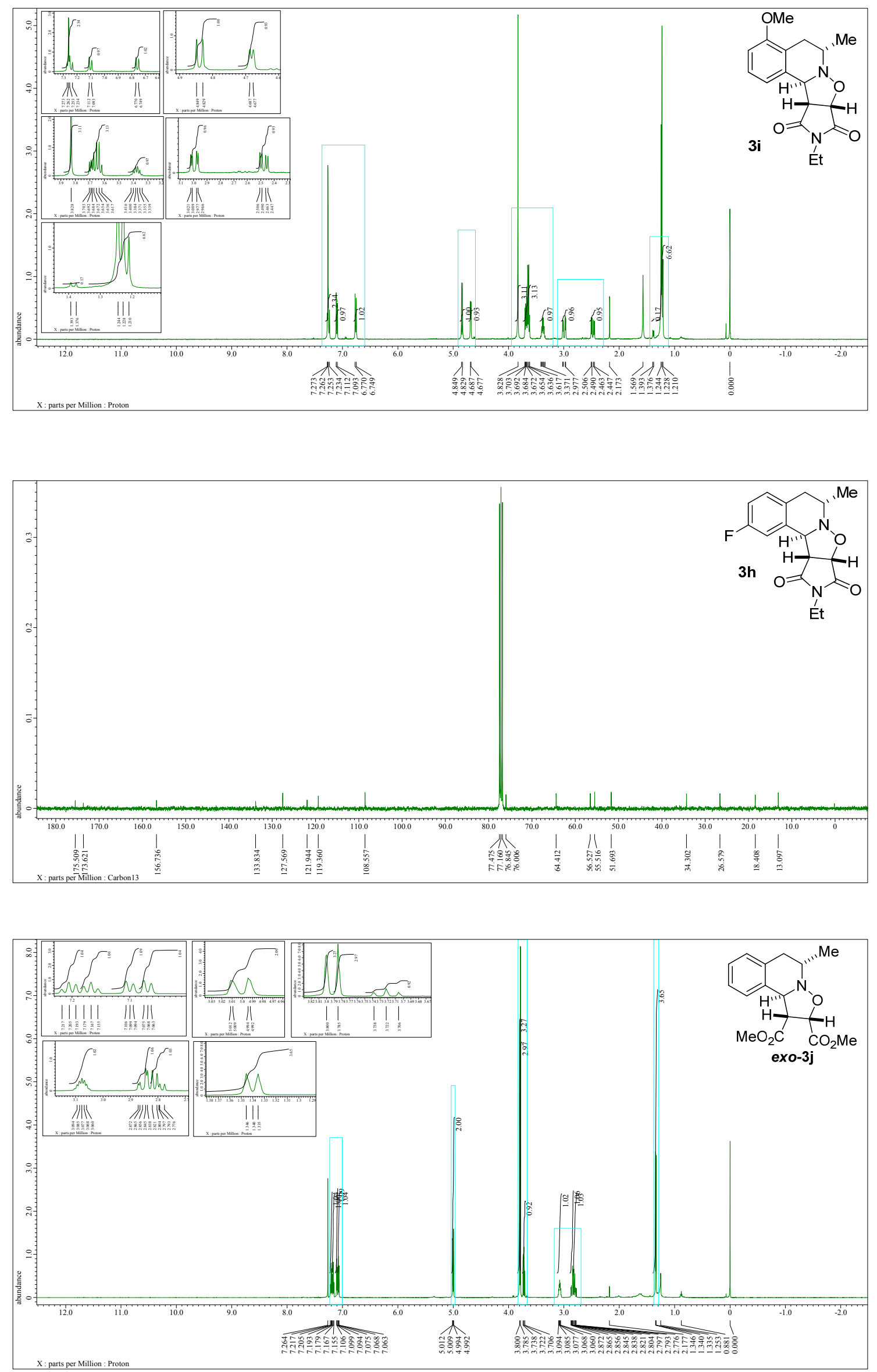

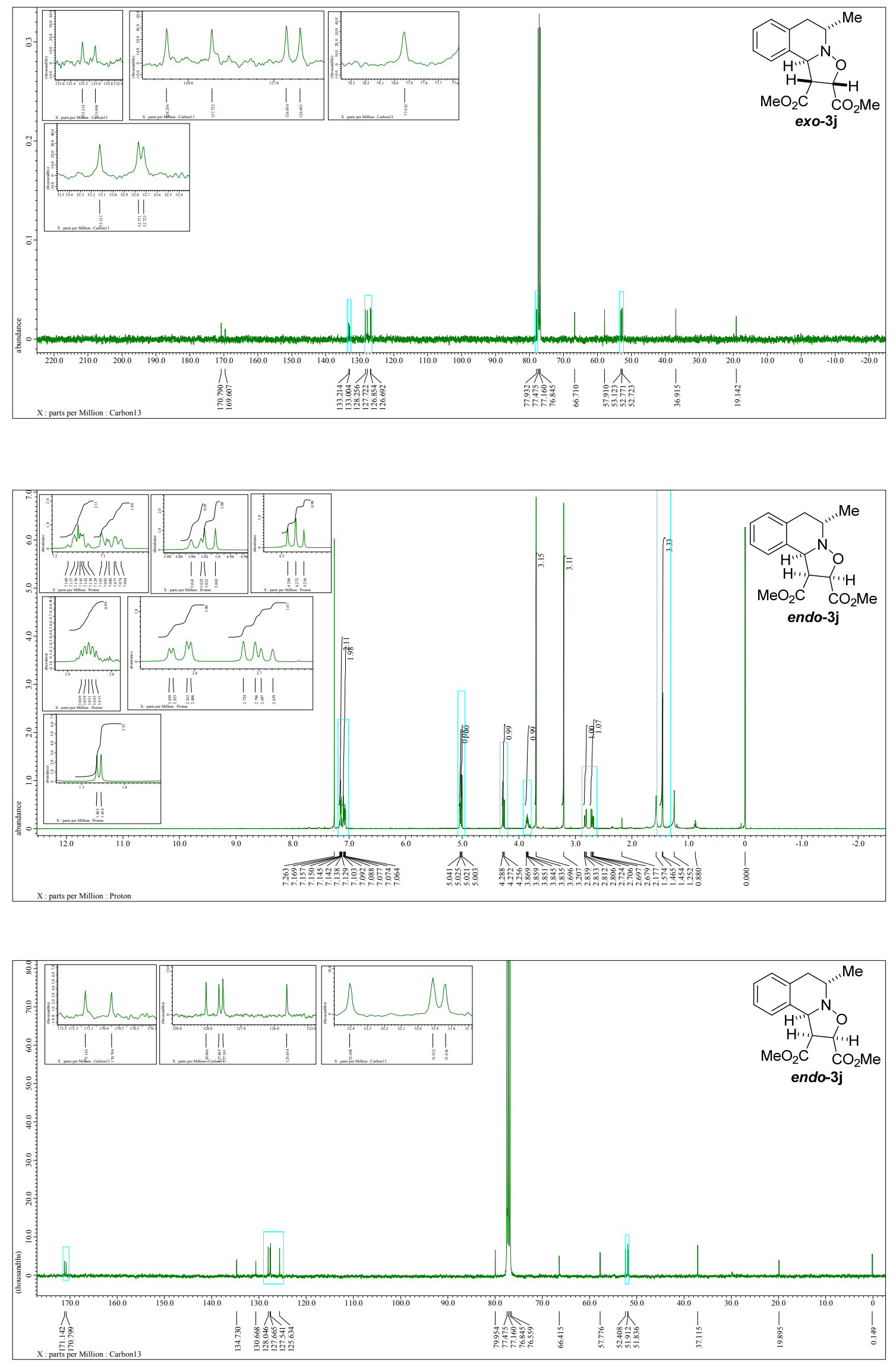


\section{G. HPLC charts}
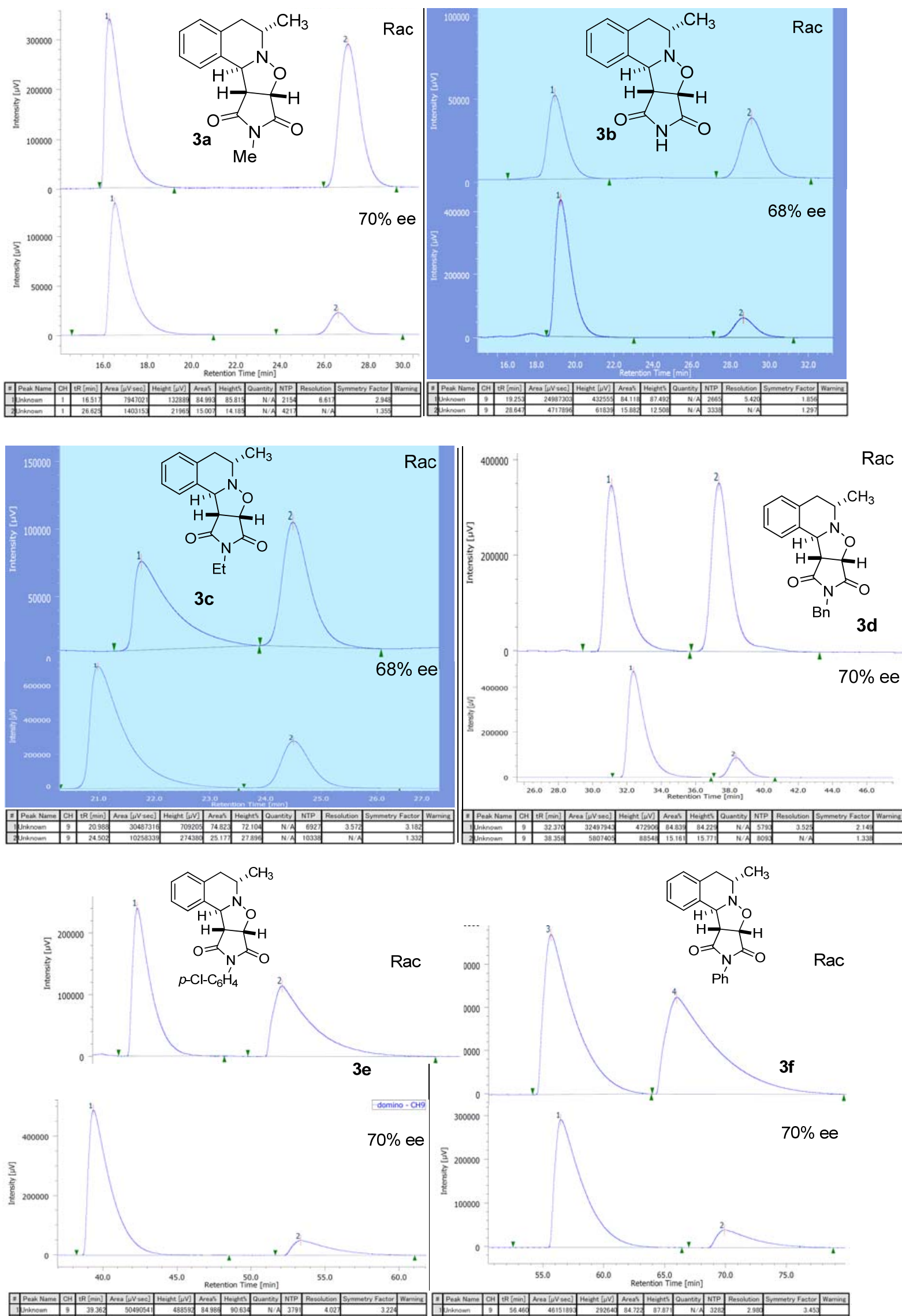

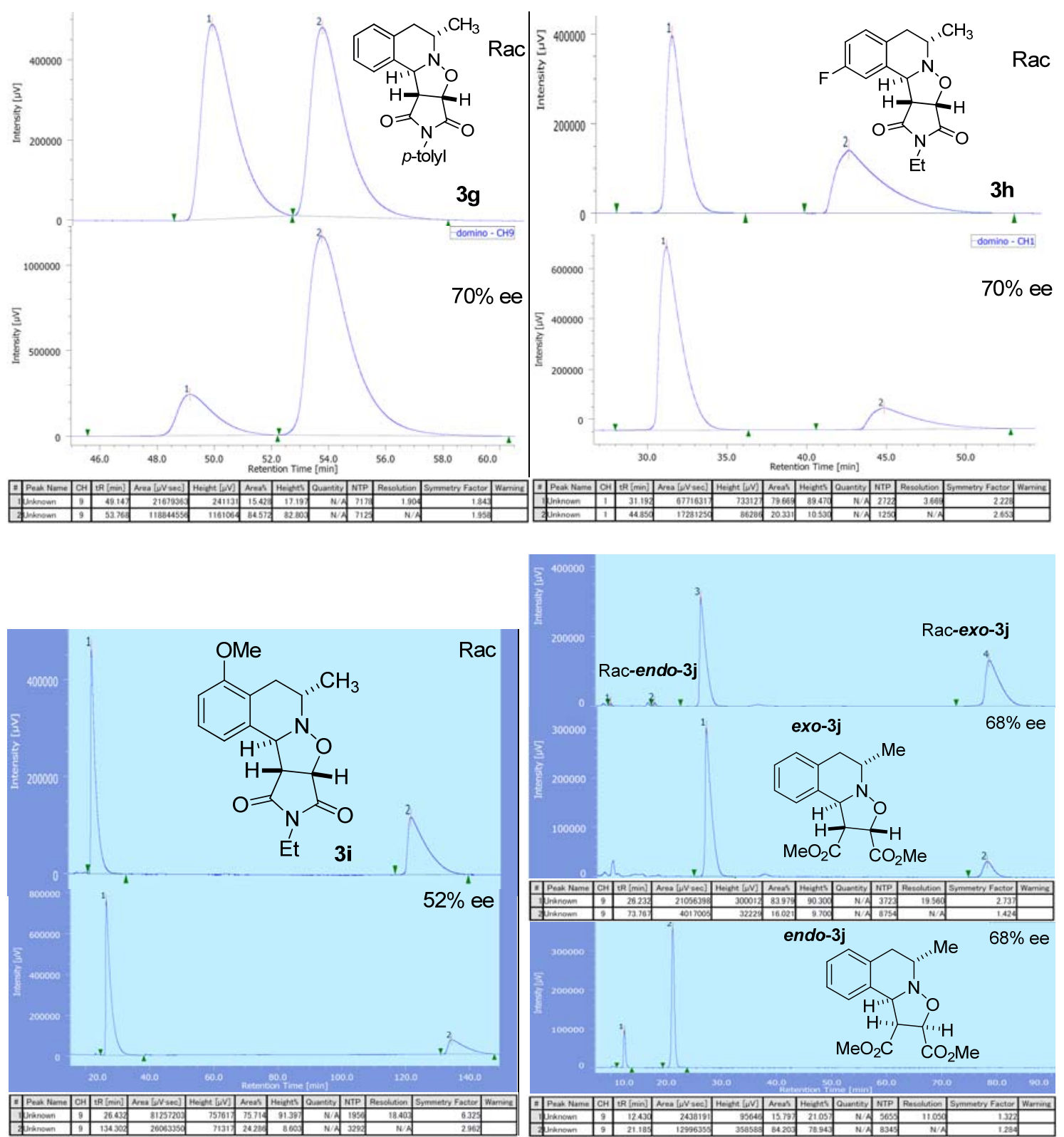